\title{
New insights into the physical processes that underpin cell division and the emergence of different cellular and multicellular structures.
}

\author{
Philip Turner ${ }^{1 *}$ Laurent Nottale ${ }^{2 \dagger}$, John $_{\text {Zhao }}{ }^{\ddagger \ddagger}$ and Edouard Pesquet ${ }^{4 \S}$ \\ ${ }^{1} 69$ Hercules Road, Sherford, Plymouth, Devon, PL9 8FA, United Kingdom. \\ ${ }^{2}$ CNRS, LUTH, Observatoire de Paris-Meudon, 5 Place Janssen, 92190, Meudon, France. \\ ${ }^{3}$ Department of Chemistry, Durham University, South Road, Durham, DH1 3LE, United Kingdom. \\ ${ }^{4}$ Stockholm University, Dept of Ecology, Environment and Plant Sciences, Stockholm, 106 91, Sweden.
}

April 9, 2019

\begin{abstract}
Despite decades of focused research, a detailed understanding of the fundamental physical processes that underpin biological systems (structures and processes) remains an open challenge. Within the present paper we report on biomimetic studies, which offer new insights into the process of cell division and the emergence of different cellular and multicellular structures.

Experimental studies specifically investigated the impact of including different concentrations of charged bio-molecules (cytokinin and gibberellic acid) on the growth of $\mathrm{BaCO}_{3}-\mathrm{SiO}_{2}$ based structures. Results highlighted the role of charge density on the emergence of long-range order, underpinned by a negentropic process. This included the growth of synthetic cell-like structures, with the intrinsic capacity to divide and change morphology at cellular and multicellular scales.

Detailed study of dividing structures supports a hypothesis that cell division is dependent on the establishment of a charge-induced macroscopic quantum potential and cell-scale quantum coherence, which allows a description in terms of a macroscopic Schrödinger-like equation, based on a constant different from the Planck constant. Whilst the system does not reflect full correspondence with standard quantum mechanics, many of the phenomena that we typically associate with such a system are recovered.

In addition to phenomena normally associated with the Schrödinger equation, we also unexpectedly report on the emergence of intrinsic spin as a macroscopic quantum phenomena, whose origins we account for within a four-dimensional fractal space-time
\end{abstract}

\footnotetext{
*pturner.physics@outlook.com

†laurent.nottale@obspm.fr

${ }^{\ddagger}$ zhengyun.zhao@durham.ac.uk

§edouard.pesquet@su.se
} 
and a macroscopic Pauli equation, which represents the non-relativistic limit of the Dirac equation.

\section{Keywords}

: Self-organization; emergence; negentropy; cell division; quantum mechanics; disorder.

Declarations of interest: none

\section{Introduction}

\subsection{General background}

Despite decades of focused research, a detailed understanding of the fundamental physical processes which govern biological systems remains an open challenge. The difficulty lies in part in deconstructing the vast, multi-scale array of systems and processes, which collectively interact to create extraordinary levels of complexity in a living organism. However it also relates to lack of consensus on the existence, nature and origin of a "missing force" and an associated negentropic process that dictates the emergence of order rather than chaos, predicted from the 2nd law of thermodynamics.

The seeds of a new approach to this challenge begin with a proposal by Prigogine et al $[1,2,3,4]$ that "many body" complex systems can be regarded as irreducibly probabilistic, in a manner analogous with Quantum Mechanics (QM). However, it is in a later programme of work $[5,6,7]$, that we begin to see the emergence of a testable theoretical framework to support such an approach. Within this new framework, biological systems are considered within the context of the theoretical foundations of QM and the debate around irreversible laws linked to determinism and probabilistic descriptions in physics.

At the very smallest scales in nature we see a fundamental irreversibility under the reflection $|d t| \rightarrow-|d t|$, which is at the origin of the complex number $\mathbb{C}$ representation of quantum mechanics, outlined in [7]. This complex representation is reflected in two real $\mathbb{R}$, irreversible processes (described by real path integrals), which when combined, result in a complex reversible system (a complex path integral), which leads to the wave function.

Decoherence of a wave packet leads to classical physics, which is at first reversible, but as time scales increase (larger than the Lyapunov timescale), classical systems, driven by irreversible thermodynamic processes, become again chaotic (irreversible). New evidence based macroscopic quantum theory [7] supports proposals in [5, 6, 8, 11], that on even longer times scales (initially predicted at $>10$ to 20 Lyapunov timescales, which we typically see in biological systems), this chaos becomes the seed for a new macroscopic quantum theory, whose equations are again reversible in terms of complex wave functions. 
The theory that macroscopic quantum processes play a key role in the emergence of structure in biological systems is supported by the growth of a range of $\mathrm{BaCO}_{3}-\mathrm{SiO}_{2}$ based, "plant-like" structures and the successful modelling of a number of these structures using macroscopic Schrödinger-like equations [7]. The work confirmed that a range of structures, typically associated with living systems, have the potential to emerge spontaneously, with different structures (and their properties) being individually dictated by a unique set of external field, limiting conditions and symmetries. However, given the preliminary nature of the work, it was not surprising that we observed a number of limitations when compared to real plants, some of which we now consider.

A key element of the work to grow plant-like structures relates to the release of protons through solvation of atmospheric $\mathrm{CO}_{2}$, which leads to ionisation of $\mathrm{BaCO}_{3}-\mathrm{SiO}_{2}$ molecules. Whilst levels of ionisation (translated as charge density), determined by $\mathrm{CO}_{2}$ concentration played a critical role in the emergence of structure, the level of control over reproducibility of structures was limited. Repeatable structures only emerged under conditions of very high or low charge density $(\rho)$. For example, an atmosphere of saturated $\mathrm{CO}_{2}$ (maximum charge) led to a monoculture of spherical, cell like structures (Fig 1b). By contrast, when $\mathrm{CO}_{2}$ was minimised by purging the atmosphere with nitrogen gas, it led to pure crystalline structures (Fig 1a), indicating that as $\rho \rightarrow 0$, molecules, unhindered by repulsive charges are permitted to form a crystal lattice. For the range of intermediate conditions between these two extremes, control of a diverse range of structures was unpredictable, with probable outcomes being only very loosely correlated with a given set of conditions. This contrasts with real biological systems where we observe precise, nuanced and robust levels of control over a wide range of conditions and subsequent structures dictated by genetic code. In translating results on the growth of inorganic plant-like structures reported in [7] to their biological equivalent we need to explain how structures are more precisely (and repeatably) controlled in real plants.
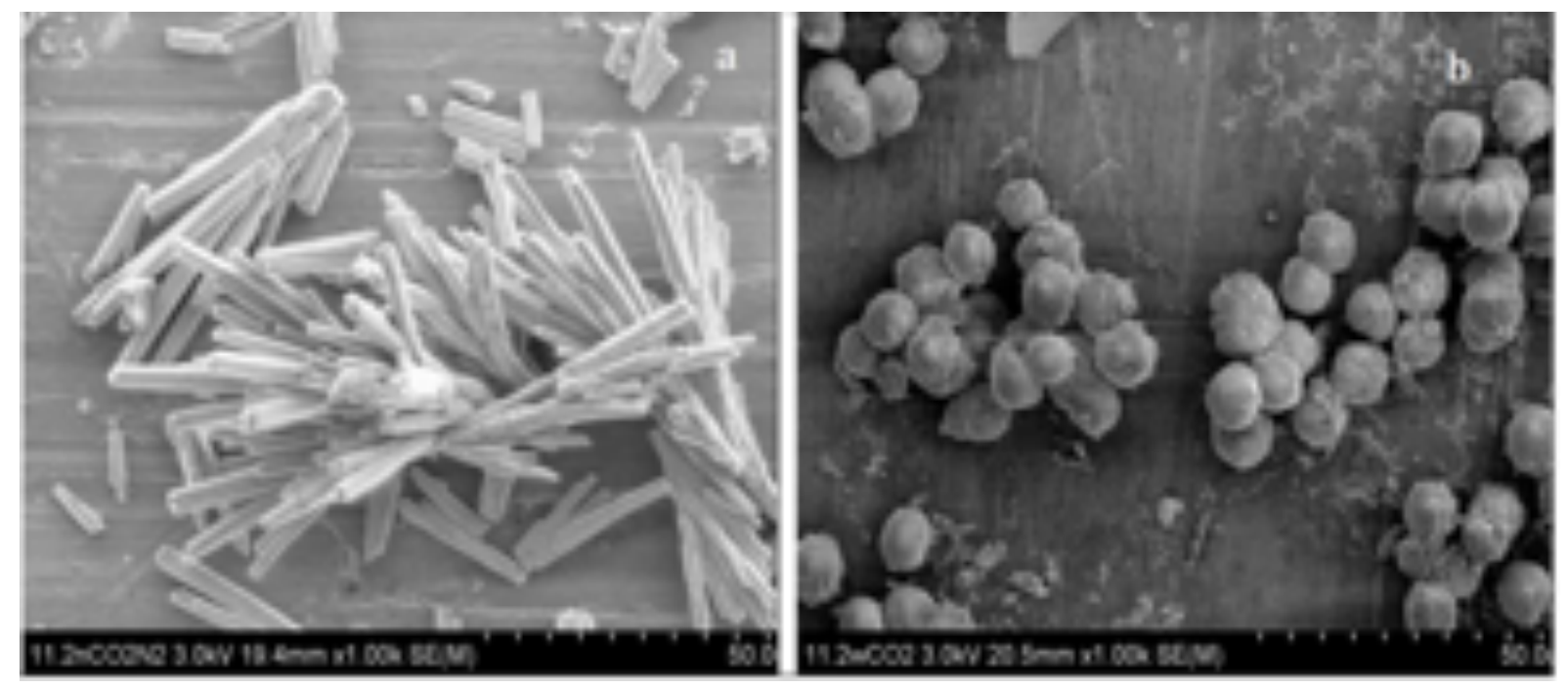

Figure 1: Crystals and cells reproduced from [7].

A second limitation in these studies related to the small-scale of the plant-like structures, 
their size being limited to the diameter range typically found in plant cells $(10-40 \mu m)$. Beyond this scale, van der Waals forces are exceeded by gravitational forces, which represent a physical constraint on cell diameter [7]. Biological systems circumvent this limitation on scale through either an increase in cell length (which has its own limitations), or more effectively through cell division to create larger scale structures. However, whilst observing the emergence of cell-like structures in [7], there was no indication of cell division and the emergence of multicellular structures under the limited set of test conditions evaluated. It was therefore concluded that further work was required to determine if it is possible to create a synthetic cell-like structure with the capacity to divide.

In order to understand the limitations linked to this earlier work [7] and how they might be resolved, we briefly summarise key theory that underpins the emergence of the macroscopic quantum systems we are considering. For a more detailed insight we refer the reader to $[7,8]$ and references therein.

\subsection{A brief review of background theory.}

The emergence of a structure such as a cell begins at the molecular scale, where quantum vacuum fluctuations alongside thermal fluctuations act as a sea of harmonic oscillators. Collectively these "environmental fluctuations", along with levels of ionisation $\rho$ have a fundamental impact on the trajectory and dynamics of particles as they interact to form larger structures. As $\rho$ increases, repulsive forces (Coulomb interactions) between adjacent charged particles, create a barrier to bonding, allowing greater freedom to interact with collective environmental fluctuations represented with a Euler equation $(\mathrm{Eq} 1)^{1}$, in which $D$ represents a standard diffusion coefficient, with the force expressed in terms of probability density $P$

$$
\left(\frac{\partial}{\partial t}+V . \nabla\right) V=-2 D^{2} \nabla\left(\frac{\Delta \sqrt{P}}{\sqrt{P}}\right) .
$$

The 'diffusion force' derives from an external potential

$$
\phi_{\text {diff }}=+2 D^{2} \Delta \sqrt{P} / \sqrt{P}
$$

which introduces a square root of probability, normally associated with quantum systems, in the description of a classical diffusion process.

At the smallest molecular scale, as levels of charge density in the system increase, charges loosely cluster, with $\AA$-scale holes within clusters creating an attractive potential well [9]. At this scale, a small cluster of charges can be seen as a quantum fluid

$$
\psi_{n}=\sum_{n=1}^{N} \psi_{n}
$$

\footnotetext{
${ }^{1}$ The detailed derivation of $\mathrm{Eq} 1$ is outlined in previous work $[7,10]$
} 
which is expected to be the solution of a Schrödinger equation

$$
\frac{\hbar^{2}}{2 m} \Delta \psi_{n}+i \hbar \frac{\partial \psi_{n}}{\partial t}=\phi \psi_{n}
$$

where $\phi$ represents an external potential or potentials, including $\phi_{\text {diff }}$ (Eq. 2).

Introducing $\rho$ and phase, defined as a dimensioned action $A$ of the wave function $\psi_{n}=\sqrt{\rho_{n}} \times$ $e^{i A_{n} / \hbar}$ along with the velocity field of the quantum fluid $(n)$, given by $V_{n}=(\hbar / m) \nabla A_{n} / \hbar$, we can rewrite the Schrödinger equation in equivalent form, as Euler (Eq.5) and continuity equations (Eq.6), which reflect the real and imaginary part of the Schrödinger equation,

$$
\begin{gathered}
\frac{\partial V_{n}}{\partial t}+V_{n} . \nabla V_{n}=-\frac{\nabla \phi}{m}-\frac{\nabla Q_{n}}{m}, \\
\frac{\partial \rho_{n}}{\partial t}+\operatorname{div}\left(\rho_{n} V_{n}\right)=0,
\end{gathered}
$$

where $Q_{n}$ represents a localized quantum potential,

$$
Q_{n}=-\frac{\hbar^{2}}{2 m} \frac{\Delta \sqrt{\rho_{n}}}{\sqrt{\rho_{n}}}
$$

which is implicit in the Schrödinger equation (Eq.4), but now explicit in the Euler equation (Eq.5).

When considering the impact of environmental fluctuations on molecular assembly beyond a small cluster of charges, successive solutions during time evolution of a time-dependent Schrödinger equation in a 2D harmonic oscillator potential (plotted as isodensities) lead to a model of bifurcation (Fig 2). Bifurcation occurs as the energy level increases from the fundamental level $(n=0)$ to the first excited level $(n=1)$ and the system jumps from a one-body to a two-body, branched structure ${ }^{2}$. Under the influence of environmental fluctuations, an iterative bifurcation process leads to the emergence of a branched molecular assembly with high levels of disorder (seen as fractal architectures).

Beyond a critical point in molecular assembly, an increase in charge density and charge disorder, linked with a fractal molecular structure, means that "valleys" between attractive potential wells merge, leading to a transition from a small cluster of charges $\psi_{n}=\sqrt{\rho_{n}} \times e^{i A_{n} / \hbar}$ (where $A_{n}$ is a microscopic action), to an interconnected, macroscopic fractal network of charge induced channels, illustrated in a simplistic, two dimensional model in Fig $3^{3}$.

This network of channels, with interconnected local potential wells acting as the roots of a fractal system, dictates the trajectory of macroscopic fluctuations in a process analogous to that observed in Coherent Random Lasing (CRL) [9]. At a critical percolation threshold,

\footnotetext{
${ }^{2}$ Whilst the model can be illustrated by an harmonic oscillator potential (2D or 3D) and by box solutions [8], it is a very general feature of solutions of the Schrödinger equation, that fundamental ('vacuum') states show a unique global structure, while first excited states generally show a two-body structure.

${ }^{3}$ Extending the scenario in Fig 3 to a 3D fractal architecture will lead to the emergence of a 3D distribution of static charges $\left(\psi_{n}\right)$ and a corresponding $3 \mathrm{D}$, charge induced fractal velocity field.
} 


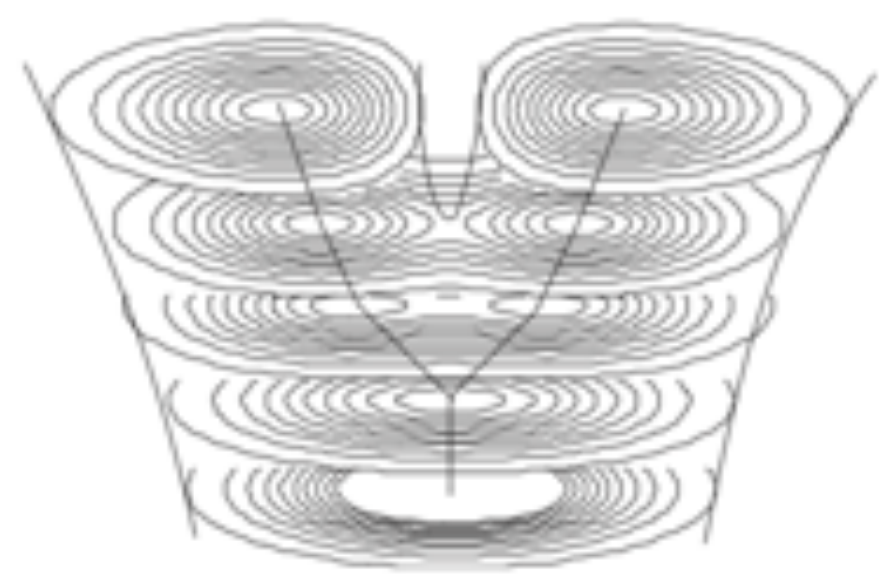

Figure 2: A model of bifurcation, described by successive solutions of the time-dependent 2D Schrödinger equation in an harmonic oscillator potential plotted as isodensities, reproduced from [6]
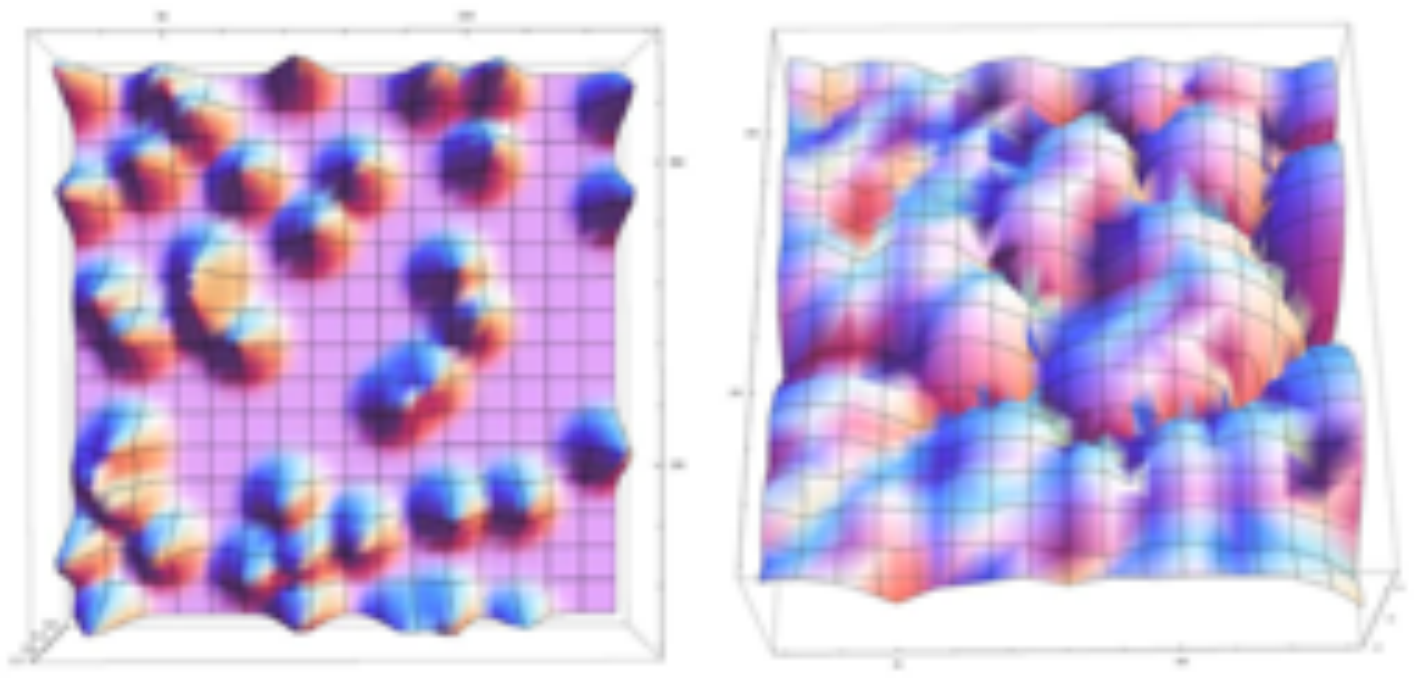

Figure 3: A disordered distribution of individual charges (left). When modelling the interaction of quantum potentials associated with each charge, they collectively create a geometric network of hills and valleys (right), reproduced from [7].

destructive interference effects cancel out of most frequencies, leaving a coherent resonant frequency, dictated by the geometry of the fractal network. The amplitude of this interconnected web of coherent charge fluctuations, represented by $\widetilde{D}$, replaces $\hbar$ as the quantum of action ${ }^{4}$, the extent of fluctuations (correlation length) being determined by the scale of the interconnected structure. This interconnected geodesic velocity field underpins a macroscopic wave function $\psi_{N}$

\footnotetext{
${ }^{4}$ In standard QM, $\hbar$ is also a geometric property of a fractal space, defined through fractal fluctuations as $\hbar=2 m \widetilde{D}[7,11]$.
} 


$$
\sum_{n=1}^{N} \psi_{n} \rightarrow \psi_{N}=\sqrt{\rho_{N}} \times e^{i A_{N} / 2 \widetilde{D}},
$$

where $A_{N}$ is now a macroscopic action.

In this macroscopic analogue of a standard quantum system, the network of interacting quantum potentials acts collectively, to create a macroscopic quantum potential,

$$
Q_{N}=-2 \widetilde{D}^{2} \frac{\Delta \sqrt{\rho_{N}}}{\sqrt{\rho_{N}}}
$$

which reflects the transformation of a classical diffusion type system, into a quantum-like system.

Taking $\psi_{N}$ and its associated macroscopic quantum potential (MQP), we can now write a macroscopic Schrödinger-like equation $(\mathrm{Eq} \mathrm{10})^{5}$, and its equivalent Euler and continuity equations (Eq's 11 and 12), in which $Q_{N}(\mathrm{Eq} 9)$ becomes explicit [7].

$$
\begin{gathered}
\widetilde{D}^{2} \Delta \psi_{N}+i \widetilde{D} \frac{\partial \psi_{N}}{\partial t}-\left(\frac{\phi}{2}\right) \psi_{N}=0 . \\
\frac{\partial V_{N}}{\partial t}+V_{N} \cdot \nabla V_{N}=-\frac{\nabla \phi}{m}-\frac{\nabla Q_{N}}{m}, \\
\frac{\partial \rho_{N}}{\partial t}+\operatorname{div}\left(\rho_{N} V_{N}\right)=0
\end{gathered}
$$

A Schrödinger-type equation is characterized by the existence of stationary solutions, yielding well-defined peaks of probability linked to quantization laws, which are themselves a consequence of the limiting (or environmental) conditions, of the forces applied and of the symmetries of the system. In other words the equation describes a universal tendency to make structures in terms of charge density $\rho$ (constructed from the distribution of geodesics), given by the squared modulus of the wave function $\psi_{N} \mathrm{Eq} 8$ [7]. This tendency to make structures allows prediction of the most probable structures among an infinity of close possibilities. The process is analogous with living systems, where morphologies are acquired through growth processes, which can be described in terms of an infinite family of virtual, fractal and locally irreversible, fluid-like trajectories.

\subsection{Decoherence of a macroscopic wave function.}

In addition to dictating a fractal assembly of charges, which leads to the emergence of a macroscopic wave function, thermal fluctuations are also responsible for its potential decoherence. An insight into the transition is revealed when comparing the diffusion system (Eq 1), with its quantum analogue (Eq 13),

\footnotetext{
${ }^{5}$ or non linear equation such as a Ginzburg-Landau-like equation [9]
} 


$$
\left(\frac{\partial}{\partial t}+V . \nabla\right) V=+2 \widetilde{D}^{2} \nabla\left(\frac{\Delta \sqrt{P}}{\sqrt{P}}\right) .
$$

Comparison between the two systems highlights a striking level of equivalence, except that in the quantum case, the diffusion constant is now complex $(\widetilde{D})$ and the quantum force, which derives from an internal quantum potential, generated by its fractal geodesics

$$
Q / m=-2 \widetilde{D}^{2} \Delta \sqrt{P} / \sqrt{P}
$$

is the exact opposite of the external diffusive potential (Eq 2).

When we consider either standard or macroscopic forms of the Schrödinger equation (Eq's 4 and 10), the external potential $(\phi)$, associated with thermal fluctuations, competes with quantum potentials $\left(Q_{n}, Q_{N}\right)$ implicit in the Schrödinger equations. When the external diffusive force exceeds the internal quantum force, it leads to a process of decoherence described by "quantum Brownian motion" $[12,13]$. During collapse of the wave function, the emergence of 'pointer states' is linked to the collapse of the fractal velocity field, to its more stable roots. These roots form the preferred set of states of an open system most robust against environmental interaction, which account for the transition from a probabilistic to a deterministic classical description [7].

An illustration of how this works in practice at macroscopic scales can be found in the emergence of a fractal structure such as a fern (a fractal leaf), which reflects partial decoherence of a macroscopic wave function [7]. In the case of a fern, it still exhibits long range order, its fractal dimension $\left(D_{F}\right)$ being determined by the strength of the residual field $\left(Q_{N}\right)$, relative to the external potential $\phi_{\text {diff }}$. However, beyond a critical point in the decline of $\rho, Q_{N}$ becomes insufficient to support the emergence of a long range fractal architecture, but is sufficient to disrupt the formation of a crystal lattice, leading to the emergence of disordered tumour-like structures, where external diffusive forces (Eq.2) dictate a chaotic morphology. Meanwhile, as $\rho \rightarrow 0$, full macroscopic quantum decoherence of the field is reflected in the growth of a crystalline lattice as illustrated in Fig 1a.

This competing system of processes is fundamental to the emergence of a cell, its division and formation of multicellular structures, and subsequent changes in morphology at the scale of the cell and multicellular structures, which we now consider.

\subsection{The emergence of a cell and its division.}

Having summarised key elements in the emergence of a macroscopic quantum system we consider how this translates at a conceptual level into the emergence of a cell with the intrinsic capacity to divide.

We start with the hypothesis that the genome plays a critical role in controlling the physical environment that leads to the emergence of structure and processes such as cell division. The 
most direct mechanism to implement this process of control is represented by transcription of genetic code into RNA then translated into a range of proteins. The collective "proteome" represents a critical source of charged macromolecules themselves but also catalysts, whose enzymatic activities in association with chelating ions (e.g., $\mathrm{H}^{+}, \mathrm{K}^{+}, \mathrm{Na}^{+}, \mathrm{Ca}^{+}, \mathrm{Cl}^{-}$ and $\mathrm{HCO}^{-}$), lead to the production of specific charged molecules, such as hormones and metabolites to control cellular dynamics and fate.

The net result is a genetically controlled, complex and dynamic landscape of ions, charged biomolecules and complex biopolymers, which collectively offer an incremental range of potential values for "net" fractional charge. The system represents a powerful toolbox, which has been selected for and refined during the evolutionary process to create biological systems with extraordinary levels of control over charge density and its spatial distribution at the scale and location where the action (growth) takes place, thus defining the physical environment that dictates the emergence of a specific structure.

This complexity contrasts with the simple proton driven system reported in [7], explaining at least in part the reduced levels of control over structure observed compared to a real living organism.

The smallest scale at which we expect a MQP to emerge begins with molecular assembly into $n m$-scale structures (ubiquitous in living systems), which then become the building blocks for larger, sub-cellular structures [7]. A hypothesis for the emergence of $n m$-scale structures, described in [7], explains how short-range electromagnetic field fluctuations associated with the quantum vacuum, play a key role in assembly (at this scale), by coupling synergistically with a charge induced complex velocity field, to create a two-component MQP and its associated structure.

In practice, the first $\mathrm{nm}$-scale of assembly which has the potential to lead to the emergence of a MQP, appears to take the form of intrinsically disordered proteins, which represent a wide range of different structures and functionalities. Interestingly, whilst disordered proteins have been reported to represent $33 \%$ of proteins in eukaryotes, they represent only $4.2 \%$ of eubacterial proteins [14]. This suggests a strong link between protein complexity and more advanced evolutionary development.

The idea of quantum criticality in proteins proposed in [7] is supported by independent theoretical studies [15], which suggest that complex protein structures have evolved as a key component of biological systems, precisely because their complex structures exhibit macroscopic quantum properties, which play a key role in biochemical electronic processes.

Most recently, macroscopic quantum coherence in proteins has been experimentally confirmed for an electrochemically inert $\approx 200 K D$ protein [16]. One interesting observation from this work was that superconductivity only occurred above a critical current threshold. This type of result was anticipated in [9], which draws a direct analogy with elevated levels of gain required to induce Coherent Random Lasing (CRL) in disordered media. In either scenario, macroscopic quantum coherence is not anticipated below a "critical gain" threshold. This new data provides strong evidence to support a review of the hypothesis, which evolved from initial work by Anderson [17], that disordered networks are (exclusively) associated with localisation and insulation. 
The idea was challenged at a conceptual level in biological systems [18] and specifically refuted in theory on high temperature superconductivity (HTSC) in [9]. Within [9], several examples are cited of how a fractal arrangement of dopants has been found to correlate with HTSC [19, 20, 21]. The first example of this type of work by Frantini et al [19] was published alongside a paper by Zaanen [22] in the same edition of Nature. In his companion paper, Zaanen refers to the unexpected nature of the findings by Frantini et al [19], suggesting that a new mathematical framework is required to explain these results. A proposal to address this challenge is described in detail in [9]. This work, alongside the paper on biological systems [7] was summarised in [23] to highlight the common links between HTSC and biological systems.

This most recent work has not yet filtered into mainstream scientific discussions. As a result, the current general scientific consensus accounts for the cautious response of the authors in [16] to their own experimental findings. As an example, the magnitude of the conductance fluctuations observed in this work was reported as "completely unexpected, and without a known explanation". It was also stated that if a quantum-critical state exists in proteins, it raises the "perplexing question of what evolutionary advantage could flow from this otherwise highly unlikely state". One of the objectives of the present work is to provide a response to this question.

Once a macroscopic quantum system in the form of a biopolymer complex such as a protein has been created, environmental fluctuations and charge density once again play a key role in the assembly of larger scale MQP's and structures through aggregation and bifurcation. However, in this larger scale of assembly, charge density $\rho$ can now be described by the spatial distribution of each individual biopolymer complex, as an average (possibly fractional) charge in the larger scale fractal network.

In practice, different proteins and hormones, alongside other sources of charge, play multiple roles in the emergence of sub-cellular and cell structures and processes such as cell division and cell differentiation. As an example, some proteins have a special role to play as building blocks to form structures such as microtubules, whilst others play a different role in dictating local charge density and its spatial distribution.

This principle is illustrated in a study of differentiating plant cells in vitro [24], which identified 605 different proteins that associate with microtubules in the narrow window of time in which secondary cell wall thickenings are deposited in tracheary elements in Arabidopsis thaliana. Through active control of gene expression, different sets of microtubule-associated proteins (MAP's) influence patterning, organization and dynamicity of microtubules, which in part contribute to form "tracks" to guide the "cellulose synthase complexe" responsible for the manufacture and deposition of cellulose micro?brils.

A second case study on the emergence of multicellular, plant structures discussed in [7], is based on evidence reported in [25], which identified gibberellic acid (GA) as a key factor (amongst many) which dictates the fractal dimension of leaves in North American Lake Cress.

The key message from these two examples is that charge density and its distribution originate from various sources, which are used to influence emergent structures from molecular to whole 
plant scales. Depending on conditions, a wide range of structures has the potential to emerge [7]. However, as stated in Section 1.1, once structures reach the scale of a cell, gravitational forces represent a physical constraint on further expansion. If the system is to continue to grow in a significant way beyond this point, it has to divide. In other words, if we are to understand the physical processes that dictate the emergence of biological structures beyond the scale of a cell, it is essential to understand the cell division process.

As a first step we consider a very generic, theoretical approach to cell division previously described in $[7,8]$. In Fig 4 we see stationary solutions of a time-dependent Schrödinger equation in a 3D harmonic oscillator potential, which acts as a rough model of duplication. Solutions show a jump from a one-body to a two-body structure, reflecting a transition from the fundamental "vacuum" level $n=0$ to the first excited level $n=1$. Solutions can only take discretized morphologies in correspondence with the quantized value of the energy $2 \widetilde{D} \omega$. As energy increases beyond the one-body case $\left(E_{0}=3 \widetilde{D} \omega\right)$, no stable solution can exist until it reaches the second quantized level at $E_{1}=5 \widetilde{D} \omega$.

An alternative representation of the cell division process is given in Fig 5, where successive figures give the isovalues of the density of probability for 16 time steps. The first and last steps $(1, n=0$ and 16, $n=1)$ are solutions of the stationary (time-independent) Schrödinger equation, whilst intermediate steps are exact solutions of the time-dependent Schrödinger equation reflecting transient structures. The model shares a number of common features with the bifurcation process in Fig 2, the key difference being that in the case of bifurcation, the previous structures remain and add to themselves instead of disappearing as in cell division. The only other difference lies in the quantum of action, with Fig 2, being based on $\hbar$ rather than $\widetilde{D}$ (Fig 5), although the bifurcation process represented in Fig 2 is also valid for a macroscopic quantum system at both sub-cellular and multicellular scales, in which $\widetilde{D}$ is substituted for $\hbar / 2 m$.

The emergence of a cell with the capacity to divide represents a critical step in addressing both the conservation of genetic information and gravitational constraints on scale through the creation of multicellular structures.

An iterative process of cell division and bifurcation at multicellular scales leads to a "tissue" of cells, whose structure is dependent on net charge density at the scale of the cell. Once again, depending on charge density and fractal distribution, we see the emergence of a wide range of potential structures including macroscopic quantum systems at multicellular scales, with the cell acting as a macroscopic quantum building block, or "quanta of life" in the same way that we have outlined for a protein complex at sub-cellular scales.

Within this interpretation, the average, fractional charge associated with a cell as a composite of $n$ macroscopic wave functions, will play a key role in dictating the emergence of a multicellular MQP which can then be inserted in a growth equation, which takes a Schrödinger form [7]. Its solution yields a new, larger scale of organization such as seeds, flowers, leaves and fruits to whole organisms, whose internal fractal structure is essential for the emergence of order out of chaos.

Whilst the cell division process as represented in Fig's 4 and 5 does not reflect the level of 


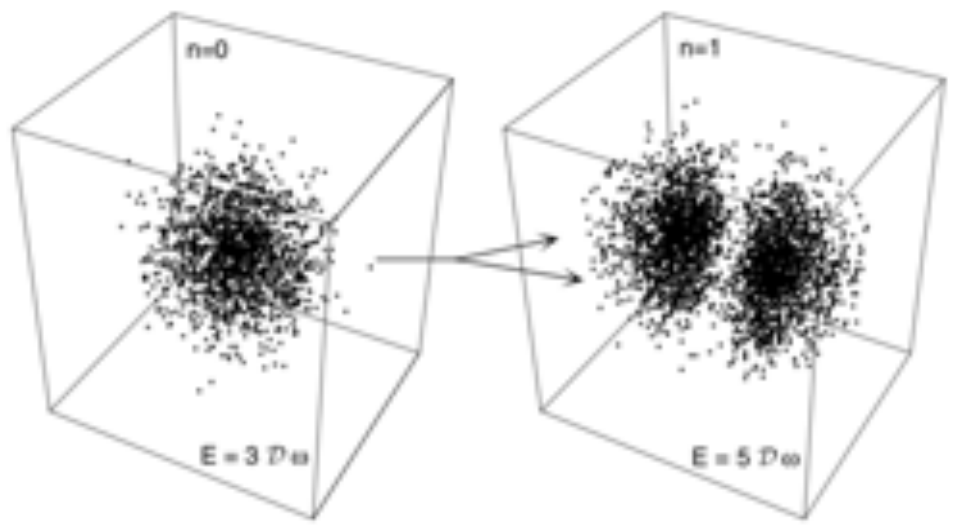

Figure 4: Model of division, reproduced from Nottale [8]. Note the revised notation with $\mathcal{D}$ being replaced by $\widetilde{D}$ in the present paper.

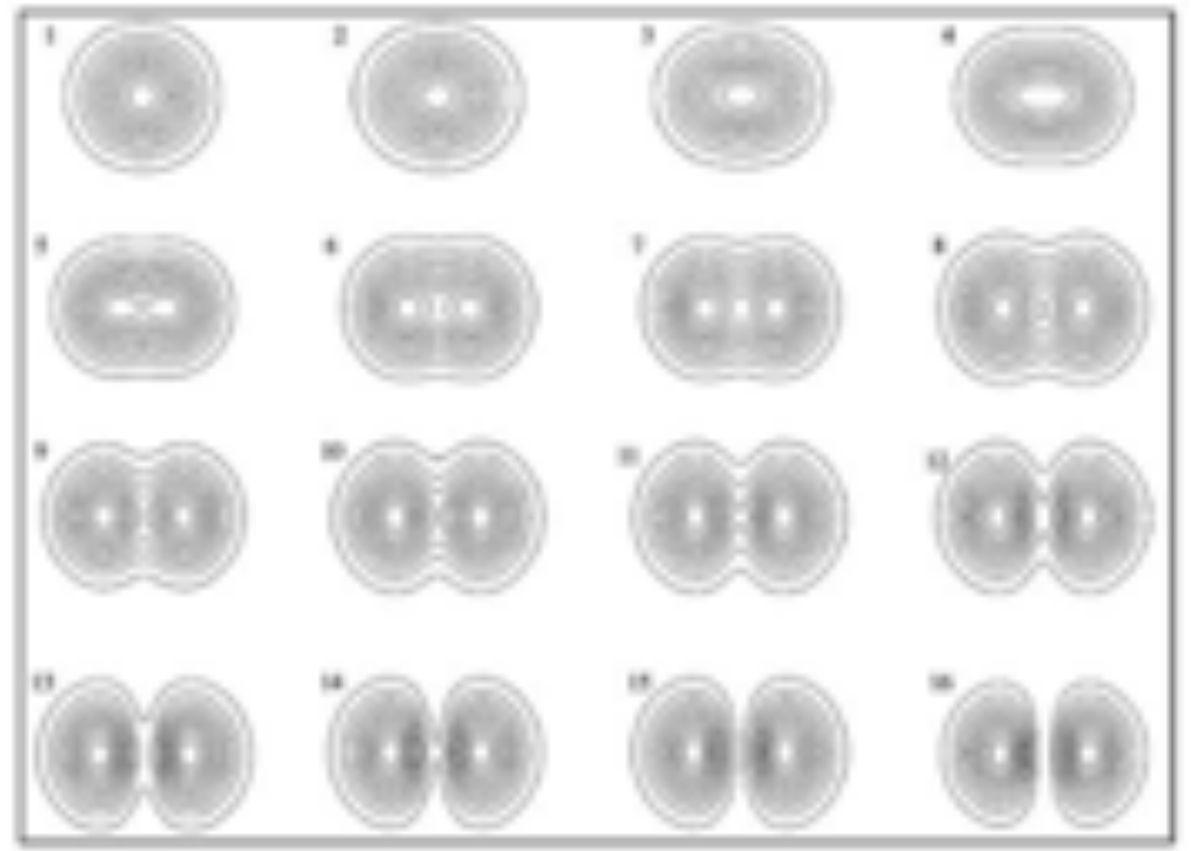

Figure 5: Model of cell division, reproduced from Auffray and Nottale [6].

complexity associated with a real biological system, the approach appears to be particularly well suited as a generic model for spontaneous duplication of quantized structures, linked to energy jumps in the presence of environmental fluctuations. The challenge of the present paper lies in beginning to fill in the gaps in our understanding of the detailed mechanism which drives the cell division process in a real biological system. 


\section{Experimental work}

\subsection{Objectives}

The extraordinary levels of complexity associated with biological systems means a full description of all variables and how they relate to the emergence of different structures and functions may take decades of detailed multidisciplinary research and analysis. However, using carefully designed inorganic growth experiments described previously [7], there is a great deal we can learn about how initial conditions can lead to specific structures and functions. A better knowledge of these systems and what governs them will improve our understanding of the mechanisms at play.

The primary objective of experimental work was to progress towards addressing limitations on control and scale of structure identified in Section 1.1, by inducing a biomimetic cell division process. Since cell division and bifurcation processes share a common physical basis (see Section 1.4), our approach was to determine if the incorporation of plant hormones (associated with bifurcation and cell division) into the $\mathrm{BaCO}_{3}-\mathrm{SiO}_{2}$ based system previously considered [7], could facilitate the growth of cell-like structures with the inherent capacity to divide and form simple multicellular structures. Hormones selected for trials included Cytokinin (CK) and Gibberellic acid (GA).

$\mathrm{CK}$ is involved in a number of plant processes, including cell division, and lateral shoot and root morphogenesis, with increased concentrations of CK leading to an increase in bifurcation.

GA is also involved in a number of plant processes including: stimulation of stem and root growth, inducing mitotic division in the leaves of some plants and increase seed germination rate. As mentioned in Section 1.4, GA has also been reported to play a role in the $D_{F}$ of leaf morphology in North American Lake Cress [25].

Since both CK and GA are at some level involved in cell division and the $D_{F}$ of plant morphology, they represented good candidates for inclusion in an experimental program with the following goals:

- Test the proposed theoretical principles underpinning the emergence of a charge induced macroscopic quantum system and its role in cell division and bifurcation at both sub-cellular and multi-cellular scales.

- Generate new insights into the detailed mechanisms underpinning cell division.

- Assess the impact of hormone concentration, as a surrogate for charge density. This mimics different values associated with a concentration gradient and local maxima in real plants, where a single hormone can induce different responses.

- Test the hypothesis that specific biomolecules under genetic control have been individually or collectively selected for in the evolutionary process to create a set of physical conditions that lead to the emergence of cell structures with the capability to divide. 
- Provide information about how CK and GA, as exemplars of charged biomolecules, influence the emergence of structure when compared with the purely inorganic, proton driven system previously reported [7, 26].

\subsection{Method}

A basic stock solution for use as an inorganic growth media was prepared following a method described by Norrduin et al [26]. It involved creating an aqueous solution of $19.1 \mathrm{mM} \mathrm{BaCl}_{2}{ }^{6}$ and $8.2 \mathrm{mM} \mathrm{Na} \mathrm{NiO}_{3}{ }^{7}$ in $200 \mathrm{ml}$ 's of freshly deionised water, mixed by continuous stirring under a nitrogen atmosphere for 30 mins. After mixing, the $\mathrm{pH}$ was adjusted with $0.1 \mathrm{M} \mathrm{HCl}$ or $\mathrm{NaOH}$ to $\mathrm{pH} 11.2$.

Two sets of formulations were then prepared from the stock solution through the addition of synthetic GA and CK (both supplied by Sigma-Aldrich), at a range of different concentrations to determine the impact of hormone concentration on the emergence of structures when compared with the original $\mathrm{BaCl}_{2}-\mathrm{Na}_{2} \mathrm{SiO}_{3}$ stock solution reported in [7].

The gibberellic acid ${ }^{8}$ Fig 6

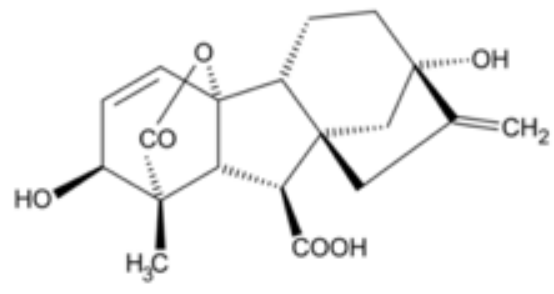

Figure 6: Gibberellic acid,

was supplied in a purified synthetic form of $\approx 90 \%$ Gibberellin $A_{3}$. With a water solubility of $\approx 5 \mathrm{~g} / \mathrm{L}$ at $20^{\circ} \mathrm{C}$, it gave a $14.43 \mathrm{mM}$ GA concentration. This was added to separate $15 \mathrm{ml}$ batches of $\mathrm{BaCl}_{2}-\mathrm{Na}_{2} \mathrm{SiO}_{3}$ stock solution to create $1 \mathrm{mM}, 2 \mathrm{mM}$ and $4 \mathrm{mM} \mathrm{GA}$ concentrations labelled $\mathrm{GA} \times 1, \mathrm{GA} \times 2$ and $\mathrm{GA} \times 4$ respectively.

A similar approach was followed for the addition of a synthetic cytokinin in the form of 6-Benzylaminopurine ${ }^{9}$

In this instance a $15 \mathrm{mM}$ batch of CK solution was prepared by adding $67.8 \mathrm{mg}$ of CK to $20 \mathrm{ml}$ deionised water in a Vulcan tube. $N_{2}$ was gently bubbled through the solution for 5 mins. The tube was then sealed and mixed by vortex at $40^{\circ} \mathrm{C}$ for 20 mins before cooling to room temperature. The solution was then added to $15 \mathrm{ml}$ batches of $\mathrm{BaCl}_{2}-\mathrm{Na}_{2} \mathrm{SiO}_{3}$ stock solution to create concentrations of $0.5 \mathrm{mM}, 1 \mathrm{mM}$ and $4 \mathrm{mM}$, labelled $\mathrm{CK} \times 0.5, \mathrm{CK} \times 1$, $\mathrm{CK} \times 4$ respectively.

\footnotetext{
${ }^{6}$ Sigma-Aldrich, MW 208.23

${ }^{7}$ Fisher scientific, MW 122.06

${ }^{8}$ Sigma-Aldrich catalogue No: G7645: $\mathrm{C}_{19} \mathrm{H}_{22} \mathrm{O}_{6}, \mathrm{MW} 346.37$

${ }^{9}$ Sigma-Aldrich catalogue No: B3408: $C_{12} H_{11} N_{5}, M W 225.25$
} 
<smiles>c1ccc(CNc2ncnc3nc[nH]c23)cc1</smiles>

Figure 7: 6-Benzylaminopurine.

Following methodology described by Norrduin et al [26], growth studies were carried out over a range of times from one hour to 5 days at room temperature $\left(18^{\circ} \mathrm{C}-25^{\circ} \mathrm{C}\right)$, on standard glass microscope slides (Deltalab, $22 \times 32 \mathrm{~mm}$ ), which were gold coated for 4 mins. For each study a slide was placed vertically in a square aluminium tank of dimensions $20 \times 20 \times 150$ $\mathrm{mm}$ (depth), containing $15 \mathrm{ml}$ of solution, which covered $\approx 30 \%$ of the height of a slide. This allowed sufficient space for structures to grow on the glass slide above the waterline. The tank was designed to operate either open or closed to the atmosphere. When closed, ambient $\mathrm{CO}_{2}$ concentration was minimised by continuous purging of the atmosphere above the solution with nitrogen gas for the duration of an experiment.

At the end of each growth period, plates were removed from solution, soaked in deionised water for $2 \mathrm{mins}$, followed by acetone for a further 2 mins. The plates were then air dried at room temperature before analysis using an Hitachi S4800 Field Emission Scanning Electron Microscope (FE-SEM) at a range of magnifications. A low voltage electron beam of $3 \mathrm{mV}$ was used to minimise potential damage to samples.

\subsection{Results and discussion}

\subsubsection{The impact of gibberellic acid (GA).}

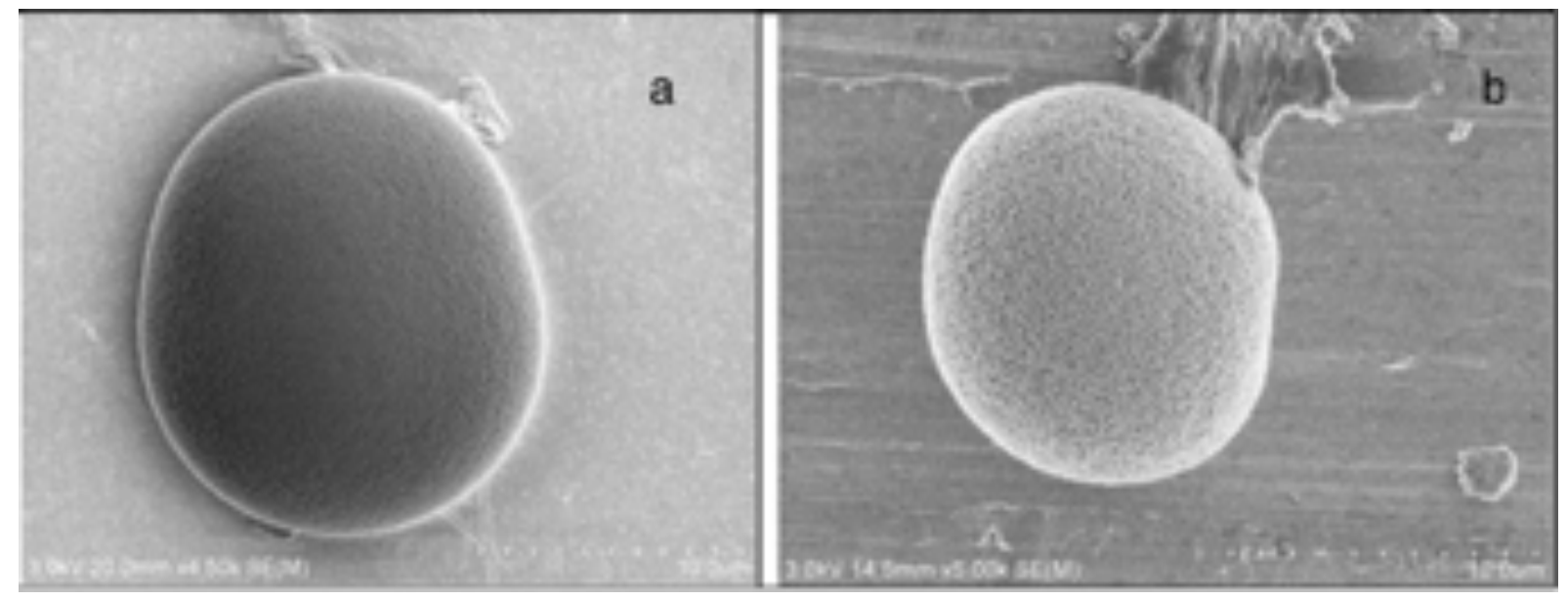

Figure 8: $\mathrm{BaCl}_{2}-\mathrm{Na}_{2} \mathrm{SiO}_{3}+\mathrm{GA} \times 1$ after 1 hour. Structure 8a was grown in air, whilst 8b was grown in an atmosphere purged with nitrogen gas. 
Fig 8a represents a typical structure emerging on the glass slide under normal atmospheric conditions, after 1 hour growth from a stock solution of $\mathrm{BaCl}_{2}-\mathrm{Na}_{2} \mathrm{SiO}_{3}$ plus $\mathrm{GA} \times 1$. The structure is composed of densely packed $(\approx 5 \mathrm{~nm}$ diameter $)$ dendrites, grown symmetrically from a centre to fill the space via an iterative process of bifurcation to create a spherical celllike structure (without a cell wall) of $\approx 15 \mu \mathrm{m}$ diameter. Due to the high density packing of the dendrites, the surface of the sphere looks relatively smooth at this magnification $(\times 4500)$.

The dendritic structure is more visible in Fig 8b, which represents a further cell-like structure of comparable diameter, grown under similar conditions, but with atmospheric $\mathrm{CO}_{2}$ (and associated protons) being purged from the system using nitrogen gas. The more open surface structure between the dendrites reflects a decline in packing density and $D_{F}$ of the dendrite network. This is explained by a reduction in charge density, associated with the purging of atmospheric $\mathrm{CO}_{2}$. The result indicates that whilst an increase in charge density associated with atmospheric $\mathrm{CO}_{2}$ leads to a higher dendrite $D_{F}$, the basic cell-like structure is defined by charge density linked to the presence of GA.

To understand this spherical cell-like structure within the context of a real biological system we need to translate the $\mathrm{BaCl}_{2}-\mathrm{Na}_{2} \mathrm{SiO}_{3} / \mathrm{GA}$ composite structures into a biological equivalence. At the simplest level we can consider these $\mathrm{nm}$-scale networks of dendrites as hydrogels, which after drying form an aerogel. Whilst there can be no direct comparison with such a simple system, the closest analogy between these cell-like structures and a real cell appears to be the dendritic contents of a protoplast, i.e., the cytoplasm, or more precisely the cytosol. In this analogy, $\mathrm{BaCl}_{2}-\mathrm{Na}_{2} \mathrm{SiO}_{3} / \mathrm{GA}$ composite dendrites act as a substitute for the dendritic structures formed by microtubules and actin filaments, which dominate the cytoplasm where most cellular activities, including cell division occur. However, we note that from a structural perspective, the cytoplasm is probably more analogous with a supersaturated hydrogel, with a good deal of unbound (free) water. In other words, the $\mathrm{BaCl}_{2}-\mathrm{Na}_{2} \mathrm{SiO}_{3} / \mathrm{GA}$ dendrite hydrogel differs from a cytosol in the sense that it offers a level of structural integrity and water holding capacity more comparable with a parenchymatic plant stem cell, in which the protoplast is encapsulated with a plasma membrane within a flexible primary cell wall composed of cellulose nano-fibrils and hemicellulose.

We stress at this point that the objective of the study was to avoid levels of complexity associated with different biological systems, by creating a simple, model structure with the potential to mimic a cell division process. Depending on the circumstances and accepting the limitations of any specific analogy, we refer to the $\mathrm{BaCl}_{2}-\mathrm{Na}_{2} \mathrm{SiO}_{3}$ hydrogel/aerogel system as either a synthetic cytosol or synthetic cell.

In both a real and synthetic cytosol we are looking at similar scales, with dendrite diameters in the 5-25 $\mathrm{nm}$ range. Actin filaments are usually about $7 \mathrm{~nm}$ in diameter, whilst microtubule filaments are normally in the region of $\approx 24 \mathrm{~nm}$ diameter, with a wall thickness of $\approx 5 \mathrm{~nm}$, created from 13 protofilaments of polymerized $\alpha / \beta$-tubulin dimers. A tubulin unit with a diameter of $\approx 5 \mathrm{~nm}$, comprised of $\approx 450$ amino acids, each with its own charge [27], creates an intrinsically disordered, complex structure with the potential to produce a MQP and macroscopic quantum coherence at the level of the tubulin unit. Evidence to support the hypothesis of macroscopic quantum coherence in intrinsically disordered structures at this scale includes Coherent Random Lasing (CRL) in nanofibres [28] and experimental 
confirmation of macroscopic quantum coherence in a protein complex [16].

A further observation relating to the combination of $\mathrm{BaCl}_{2}-\mathrm{Na}_{2} \mathrm{SiO}_{3}+\mathrm{GA} \times 1$, grown under normal atmospheric conditions, was a repeatable set of structures. A typical example of this is illustrated at $300 \times$ magnification in Fig 9 . The image reveals a monoculture of spherical objects in the $10-20 \mu m$ diameter range, which supports the hypothesis that charged biomolecules have the potential to address the uncontrolled variation in emergent $\mathrm{BaCl}_{2}-\mathrm{Na}_{2} \mathrm{SiO}_{3}$ structures previously reported in [7]. A second point relating to Fig 9 is that some cells appear to be captured in a process of division, resembling stage 10 or 11 in Fig 5.

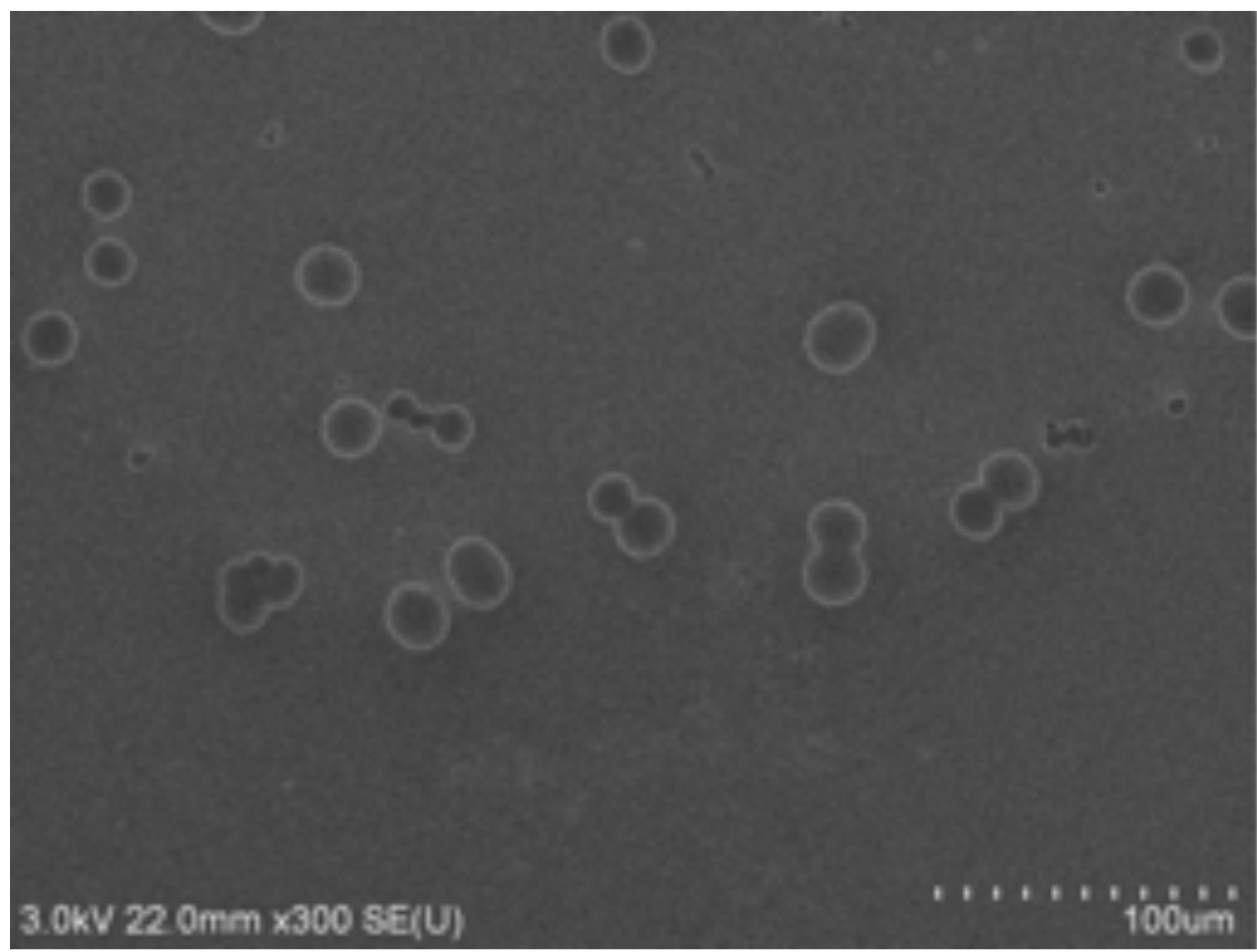

Figure 9: A monoculture of spherical structures with evidence of a division process.

To test if this "division" process was real, rather than an example of "aggregation" of preformed cellular units, the experiment was repeated in an attempt to observe additional, intermediate steps that would support the hypothesis of a division process. Examples of images captured during this additional work are reported in Fig 10 at $1200 \rightarrow 2400 \times$ magnification. The images represent a broader range of intermediate steps in the division process in Fig 5. Beyond the single body structure in Fig 10a, Fig's 10b-10e highlight subsequent steps in the transition from a 1-body to 2-body structure, delineated by an increasingly visible cleavage furrow, before complete separation in Fig 10f.

These images appear to reflect a genuine division process, representing the transitory nature of intermediate steps in the inorganic system, which not only relates to the theoretical model proposed, but also mimic a real biological system. The result lends considerable weight to the 
hypothesis of cell division as a macroscopic quantum process in which the energy associated with higher levels of quantization $(2 \widetilde{D} \omega)$ is linked to increasing $\rho$ as $[\mathrm{GA}]$ increases. Beyond a critical point, division of the cellular unit is essential to support ongoing growth of the system.

The transition from a one-body to a separate two-body system, as shown in Fig 10F, reflects the type of division we would typically expect in a single celled organism. However, in multicellular organisms, many cells remain connected after division. Given an appropriate level of charge density, this will lead to a process of bifurcation as illustrated in Fig 2, but now at the scale of a multicellular structure.

We note that the same conditions that underpin Fig 10, also led to a limited number of examples of multicellular structures. Fig's 11a-c show 3, 4 and 5-body structures after 1 hour. However, as shown in Fig's 11d-f, by increasing the period allowed for growth to 3 hours at GA $\times 1$, a further increase in cell count was observed, with Fig 11f showing the beginnings of a distinct bifurcation process at the cellular scale.

Taking this bifurcation process to its logical conclusion, the fractal dimension of a multicellular structure (which dictates cell packing density), and the average (fractional) charge on each cell, collectively dictate charge density $\rho$ and its spatial distribution. We suggest that given the right conditions, these combined factors have the potential to dictate the emergence of a multicellular MQP.

Whilst Fig's 9-11 show evidence of cell division, the images do not reveal the level of detail required to elucidate the mechanism that underpins the division process. However, a clearer picture is revealed in Fig 12. After 1 hour growth at double the previous GA concentration $(G A \times 2)$, the jump from a one-body to a two-body structure, captured in transition helps clarify the mechanism underpinning the quantized approach proposed in Fig 4.

On the one hand, like Fig 4, which reflects the stationary solutions of a time-dependent Schrödinger equation in a 3D harmonic oscillator potential, Fig 12 reflects a point-like probability density. However the two-body system in Fig 12 is also connected by a bundle of $\mathrm{nm}$-scale dendrites, which evolves in time as a fractal fluid of geodesics, within a framework of dynamic, environmental fluctuations. In other words, Fig 12 illustrates how the two body model translates into a real division process, supported by bifurcation and intrinsic disorder at the molecular and $\mathrm{nm}$ scales to create a coherent, charge induced macroscopic quantum potential, with the inherent capacity to divide at a critical (gravity induced) point of instability. Within this context, the image suggests a single interconnected system of coherent fluctuations in which at each point in the initial one-body system, trajectories exist that connect the two-body structures, until it's final separation. Under this hypothesis, it seems worthwhile to consider the possibility that within multicellular organisms, some specialised cell types have the potential to maintain some form of "intracellular coherent state", which could explain the observation of macroscopic quantum coherence reported in biological systems such as photosynthesis [29, 30] and the avian compass [31]. This level of interconnectedness both within and between cells, also offers a new perspective on the possible quantum nature of neuronal activity associated with brain function. However, the complex nature of this subject falls outside the scope of the present paper and will be consid- 


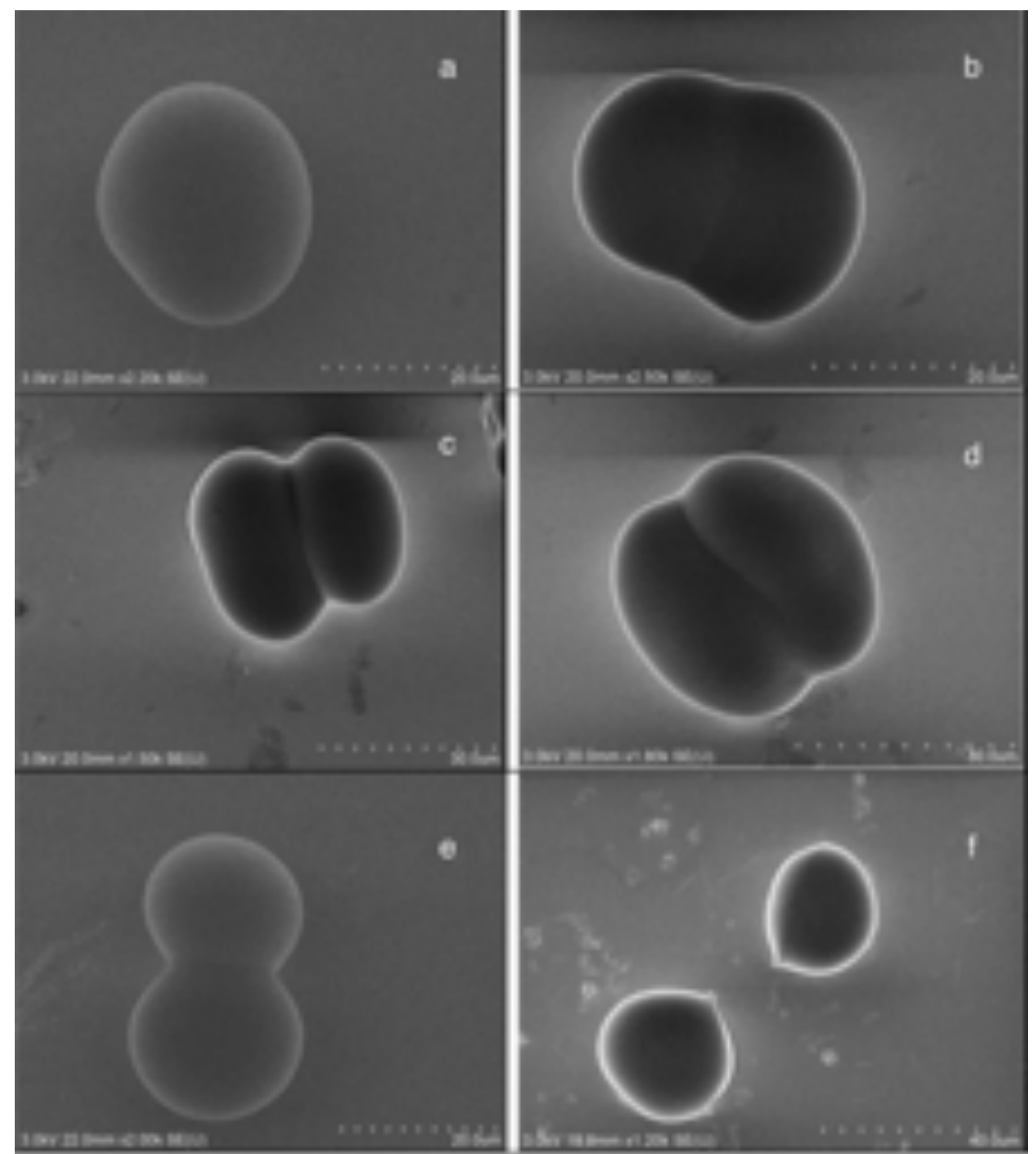

Figure 10: Different stages of a cell division process at GAx1.

ered in detail in future work. Irrespective of the outcome of the debate on neuronal activity, the intriguing implication of a growing body of evidence is that macroscopic quantum coherence is not just fundamental to cell division but may represent a defining feature of a living system.

Following on from Fig 8b, where the system was purged of atmospheric $\mathrm{CO}_{2}$ using nitrogen, Fig 13 shows further examples of a division process under the condition $G A \times 1$, in which the system was purged with nitrogen. As in the earlier case (Fig 8b), a more open dendritic structure was observed. Fig 13a illustrates an example of an early stage in the transition from a one-body to a two-body structure similar to Fig 10c, whilst Fig's 13b and 13c, which are closer to Fig 12, show a cell-like structure clearly separating into two halves. 


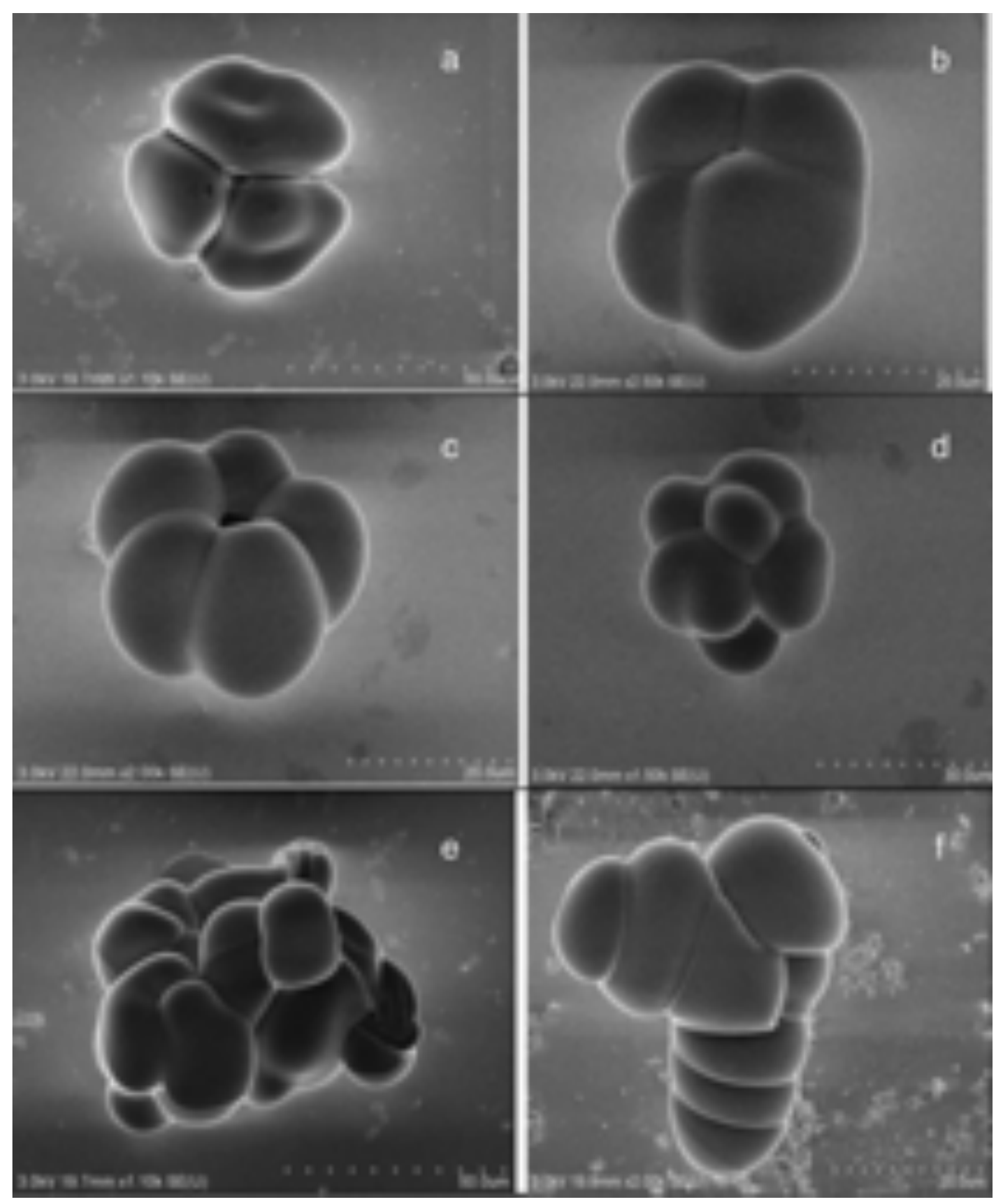

Figure 11: The emergence of multicellular structures in the cell division process after 1 hour (11a-c) and 3 hours (11d-f) at GAx1.

The transitionary, interconnecting structure in Fig 13c is more clearly revealed at higher magnification in Fig 13d, before evolving into a more advanced stage of separation in Fig 13e, 


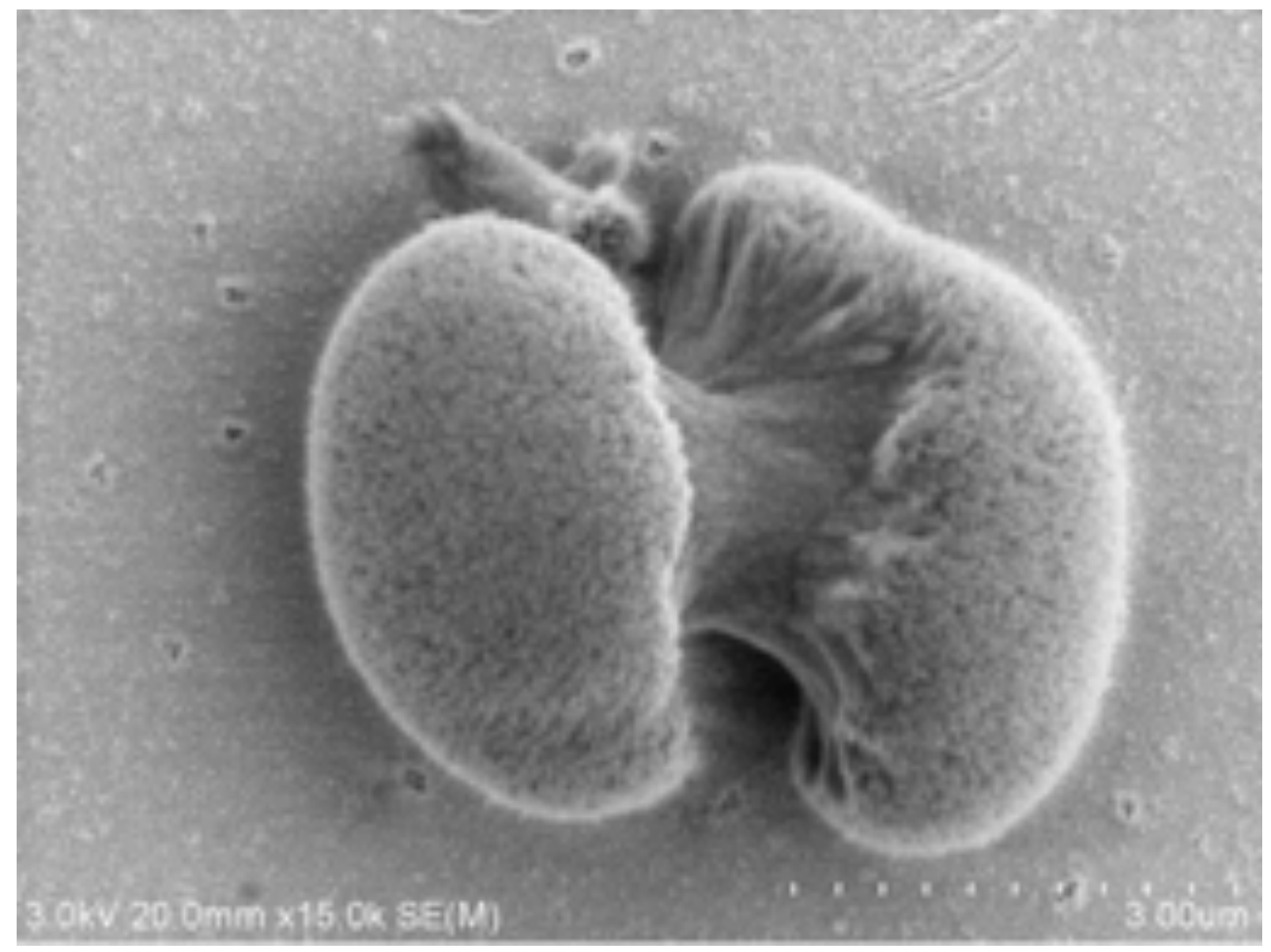

Figure 12: The process of cell division under GA $\times 2$ conditions. In order to grow beyond a critical point, a symmetric one-body structure, composed of a fractal fluid of molecular trajectories becomes unstable and is forced to divide. Here frozen in time, we see an unstable intermediate step in the dynamic transition from a one-body to a two-body structure.

which is closer to stage 13 or 14 in Fig 5. Meanwhile in Fig $13 \mathrm{f}$ we see the emergence of a six-body structure.

Fig 13 shows that despite the lower $D_{F}$ linked to a reduction in charge density, GA on its own is capable of inducing cell division, indicating that a range of values for charge density and $D_{F}$ can be effective in creating an MQP and inducing macroscopic quantum coherence. This observation has its equivalence in the variation in critical temperature (linked to macroscopic quantum coherence) with doping levels (charge density) observed in HTSC, which surround a peak critical temperature at optimal doping [9, 23].

In Fig 14 we see a further doubling of [GA] to $4 \times$ GA. Under these conditions cell duplication was a more frequent event compared with lower [GA], confirming that charge density has an important impact on the cell division process. Fig 14a shows the start of a string of cellular structures, with a fractal surface that reflects the disordered internal structure, which is revealed in a fracture in Fig 14b. Meanwhile, in Fig's 14c-f we see an increase in cell count and greater bifurcation of the cellular structure compared to multicellular structures at 


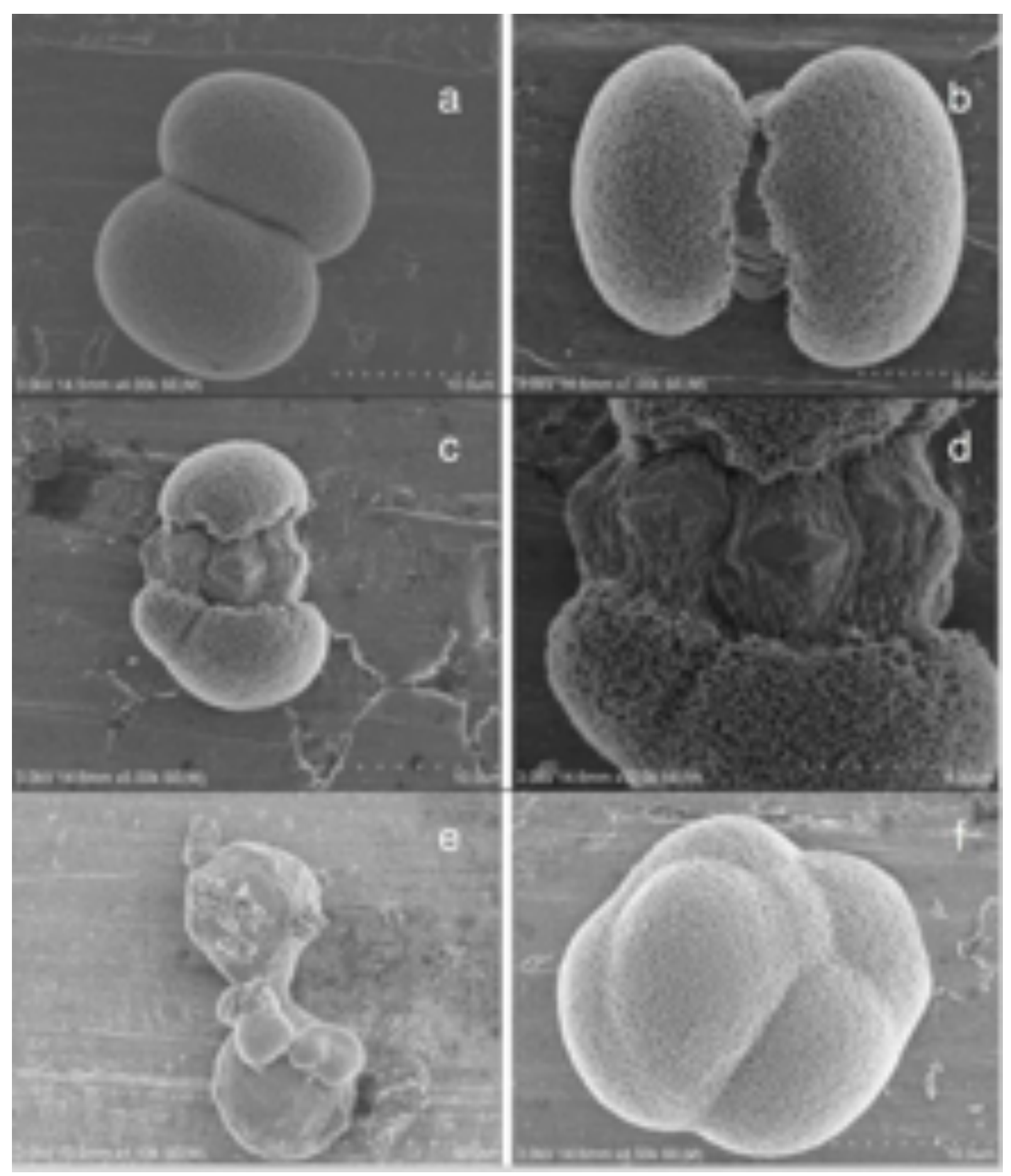

Figure 13: A cell division process observed at GA $\times 1$ in an $N_{2}$ atmosphere.

GA $\times 1$ in Fig 11. This is expected as charge density increases at the multicellular level.

It is interesting to note that the multicellular structures observed in Fig's 14c-d are remarkably similar in scale and form to stem cell division in Arabidopsis thaliana, an example of which is shown in Fig. 15. 


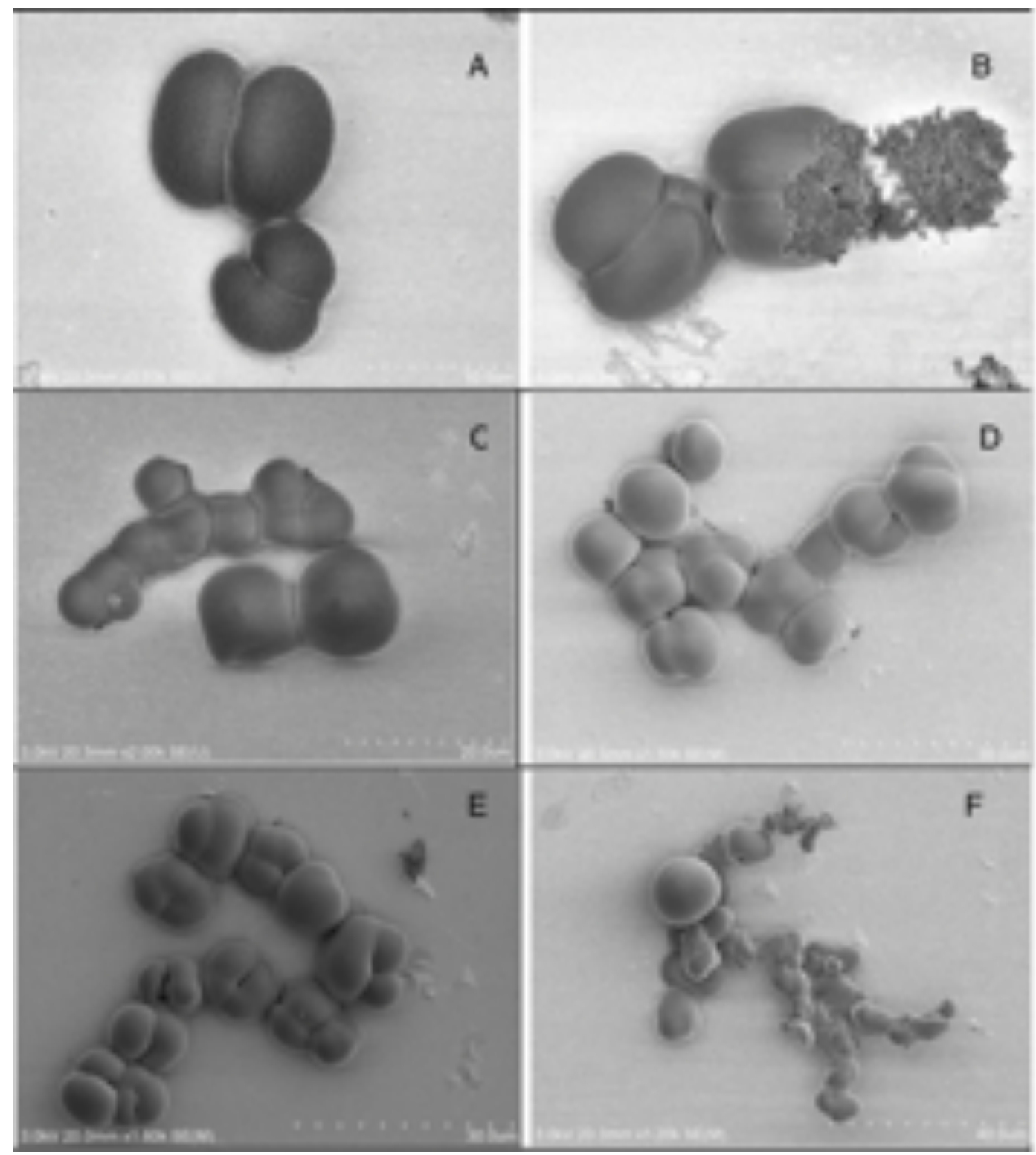

Figure 14: Examples of cell division at higher GA concentration (GAx4).

A further point of interest relates to a more ordered lattice structure between dividing bodies in Fig's 13b-13d. It suggests that flow between the two bodies during division becomes laminar, leading to a transitory lattice-like structure. Its transitory nature is illustrated in Fig 13e where the laminar zone is in decline in the later stages of division. However, whilst the disordered packing of the dendrites realigns to take on a more ordered, nematic structure during part of the transition phase, it does not necessarily exclude molecular disorder and coherence within individual dendrites. As discussed in [7], the small scale of these $n m$-scale dendrites leads to the emergence of a separate, disorder induced macroscopic wave function facilitated by quantum vacuum fluctuations.

A fractal network of charged biomolecules (and therefore charge density distribution) in the 


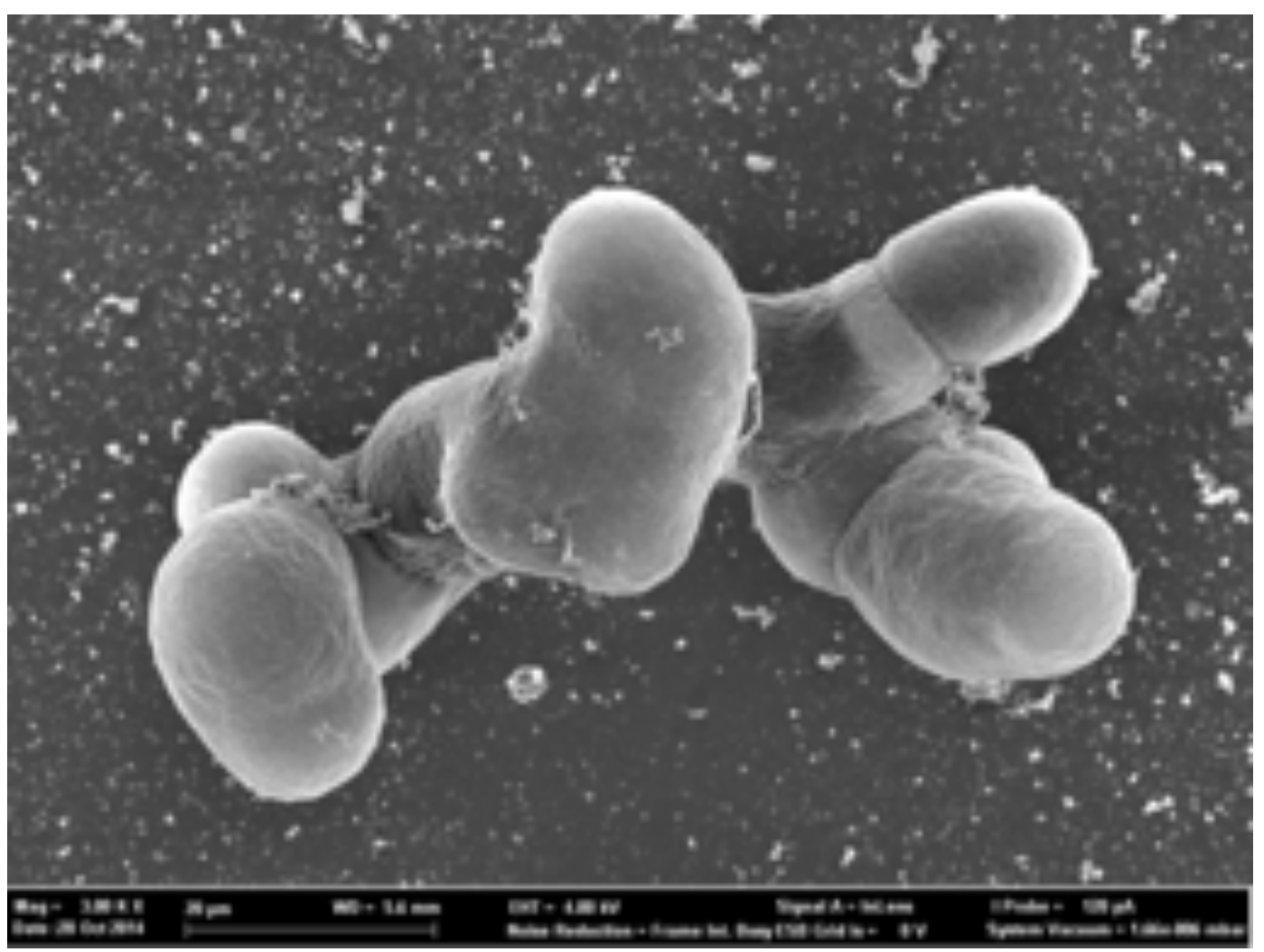

Figure 15: An example of dividing stem cells in Arabidopsis thaliana.

cytosol is key to the emergence of a macroscopic quantum potential and macroscopic wave function that can support the cell division process represented in Fig's 4 and 5 . There appears to be a strong physical equivalence between the cytosol in real cells filled with $\mathrm{nm}$ scale protein filaments (microtubules and actin filaments) and those in Fig 8-14 constructed from $\mathrm{BaCl}_{2}-\mathrm{Na}_{2} \mathrm{SiO}_{3} / \mathrm{GA}$ composite filaments.

A key difference between real and synthetic cells observed in this preliminary work with GA is the lack of a phospholipid bilayer and primary cell wall associated with real plant stem cells such as Arabidopsis thaliana illustrated in Fig 15. We therefore conclude that neither structure is required to support a basic cell division process. However, although we did not see cell wall growth under these specific conditions, we have observed the growth of an equivalent of a primary cell wall in previous work under different conditions, which is illustrated in Fig 1b in [7]. This highlights the point that different sets of conditions lead to different structures within the cell, which need to be described by separate wave functions. When modelling the emergence of structures using a Schrödinger equation, the fluid-like network of the fibrils that represents a basic cell structure can be described by one equation, whilst further equations describe the cell wall (or walls) of the cell, organelles, nucleus etc. We will consider this in more detail in Section 2.3.3. 


\subsubsection{The impact of cytokinin (CK).}

Considering $\mathrm{CK} \times 1$ after 1 hour's growth (Fig 16), the process of cell division appeared more active and advanced when compared with $\mathrm{GA} \times 1$ (Fig 11). Whilst examples of early stages of a division process were recorded (Fig 16a and 16b), results were dominated by multicellular structures, such as those illustrated in Fig's 16c-f, which are similar to Fig's 11a-e.

Alongside structures represented in Fig 16, examples of cells with growth following a righthanded, spiral trajectory, equivalent to the spiral shell of a gastropod (Fig's 17a and 17b) were observed after one hour growth at $C K \times 1$. Comparing these spiral cells with results under the same conditions after six hours (Fig's 17c-17f), there was (as with $G A \times 1$ after three hours), a notable increase in cell count in multicellular clusters. In some instances we observed clusters of the type shown in Fig's 17c and 17e. However, we also observed examples such as Fig 17d, in which individual cells exhibited a left-handed spiral similar to Fig's $17 \mathrm{a}$ and 17b. In addition, as illustrated in Fig 17f, a number of examples of extended, spiral structures $\approx 100-200 \mu m$ in length were also observed.

Note that the diameter of the helix in Fig $17 \mathrm{f}$ is of the same scale as Fig $17 \mathrm{a}(\approx 15 \mu \mathrm{m})$, but shown at a lower, $400 \times$ magnification (compared to $3500 \times$ magnification in Fig 17a) to reveal the full length of the helical structure. Note also that the helix in Fig $17 \mathrm{f}$ appears to be composed of two parallel structures forming a double, right-handed helix. Whilst at this stage we cannot be certain, one possible interpretation is that the double helix emerged after a division process in which two interconnected cells (e.g. Fig 16b), subsequently grew in a synchronous spiral trajectory. However there is an alternative interpretation to this idea, which is discussed in detail in Section 2.3.6, once we have considered the theoretical origins of spin in Sections 2.3.4 and 2.3.5.

At this point we reflect on possible mechanisms for growth once a system reaches the scale of a cell. Considering the limitations of scale imposed on cell diameter by gravitational forces, the first option is to divide and separate (in the case of a single celled organism), or form multicellular organisms. The second option for growth is for the cell to differentiate without increasing the diameter of the primary cell structure. The simplest process involves an increase in cell length. However, as seen in the diversity of cell types in the natural world, there are a large number of potential options for cell differentiation, of which the spiral structures observed in the current study represent just one scenario.

It is useful to attempt to translate these spiral structures into observations in living systems and determine if they can be explained in terms of a macroscopic quantum process. In terms of scale, the closest analogy to the spiral structure in Fig 17f, appears to be the single celled spiral diatoms, with the cell walls of an example such as Chaetoceros debilis made of silica. We also see spiral secondary cell wall structures at the same scale observed in Fig $17 \mathrm{f}$ in larger plants. For example, Fig 18 reveals a series of FE-SEM images from a stem of Arapidopsis thaliana after 12 weeks growth. At $2500 \times$ magnification (Fig 18a) we see a 100 $\mu m$ length section of spiral thickening, lining the primary wall of tracheary elements. At the same magnification, Fig 18b reveals spiral thickening from the outside of the cell, alongside larger $5000 \times$ and $10000 \times$ magnifications in Fig's 18c and 18d. A greater level of detail 


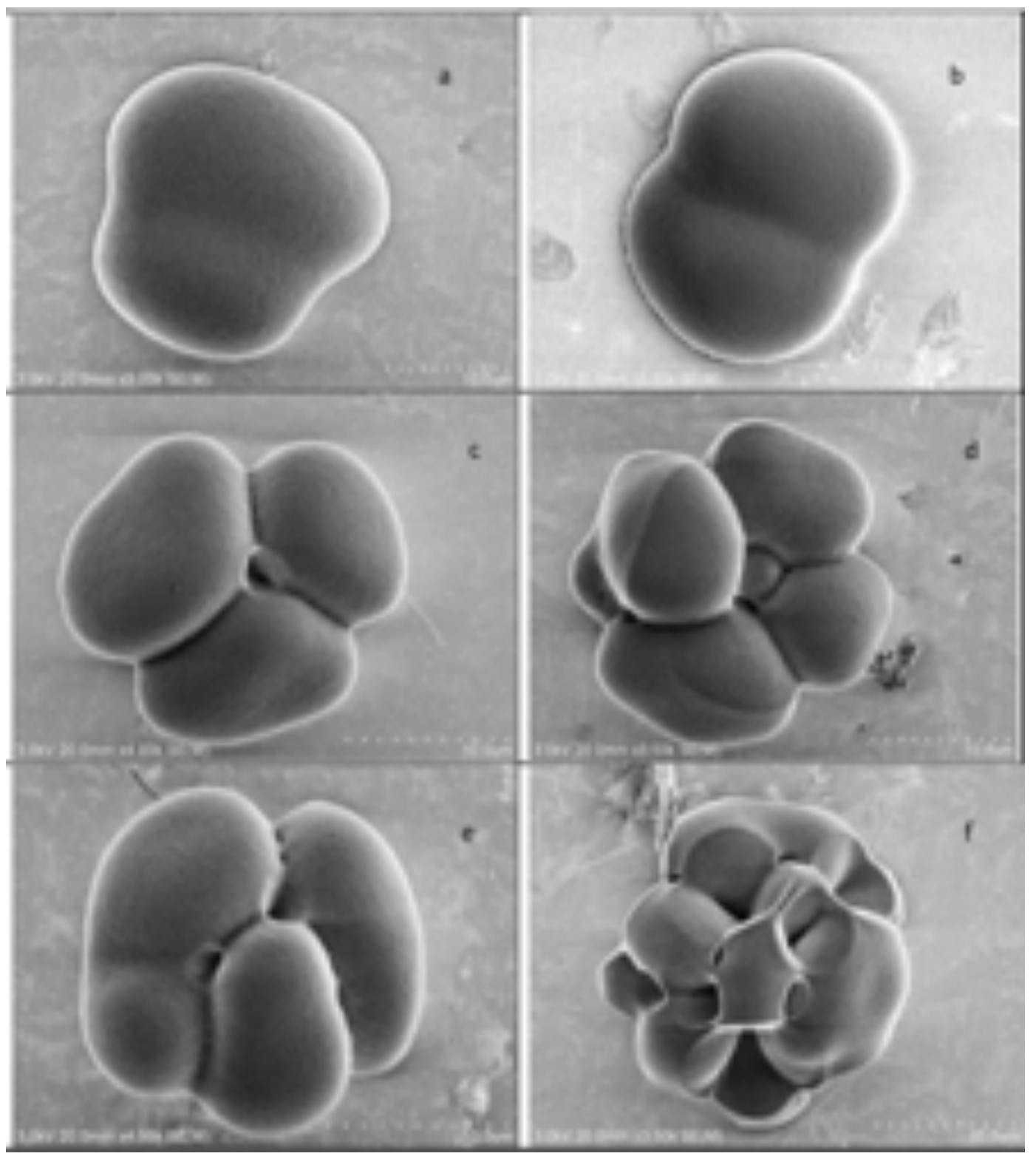

Figure 16: Examples of cell division after 1 hour at $\mathrm{CK} \times 1$.

is confirmed in Fig $18 \mathrm{e}$ at $1000 \times$ and Fig $18 \mathrm{f}$ at $5000 \times$ magnification, where a black circle highlights the spiral nature of the cell wall structure.

The observation of "spin" in the present work has interesting implications for the development of a more comprehensive macroscopic quantum theory. As highlighted earlier in the present paper and more in depth in [7], within the context of simple, nondifferentiated stem cell-like structures (e.g. Fig 8), we associate fractal, non-differentiable geometry with symmetry breaking $d t \rightarrow-d t$, which leads to a doubling of the total derivative $d / d t \rightarrow\left(d_{+} / d t, d_{-} / d t\right)$. We accommodate this two-valuedness through algebra doubling, jumping from real numbers $\mathbb{R}$ to complex numbers $\mathbb{C}$. Reversibility is recovered at the level of the wavefunction and the Schrödinger equation, which is applicable at both microscopic 


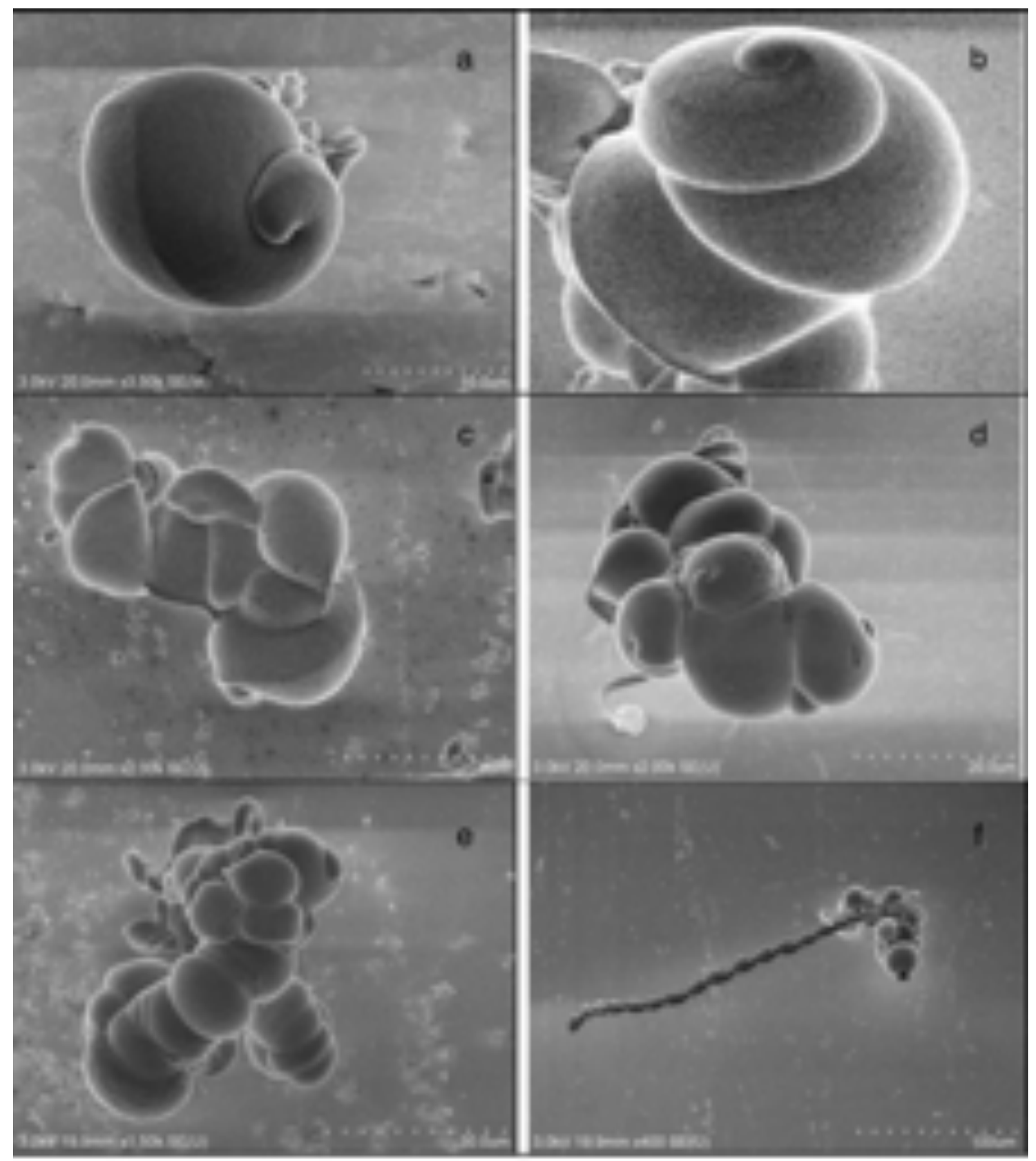

Figure 17: Cell spin and division after 1 hour (a-b) and 6 hours (c-f) at $\mathrm{CK} \times 1$.

(Eq. 4) and macroscopic scales (Eq.10). However, only bosons are coherent at macroscopic scales $[7,9,23]$. Whilst this limits the quantum properties of a macroscopic system, many of the phenomena associated with standard quantum theory are recovered, including quantization, non-dissipation, self-organization, confinement, structuration conditioned by the environment, environmental fluctuations leading to macroscopic quantum decoherence and evolutionary time described by a time dependent Schrödinger-like equation, which describes 

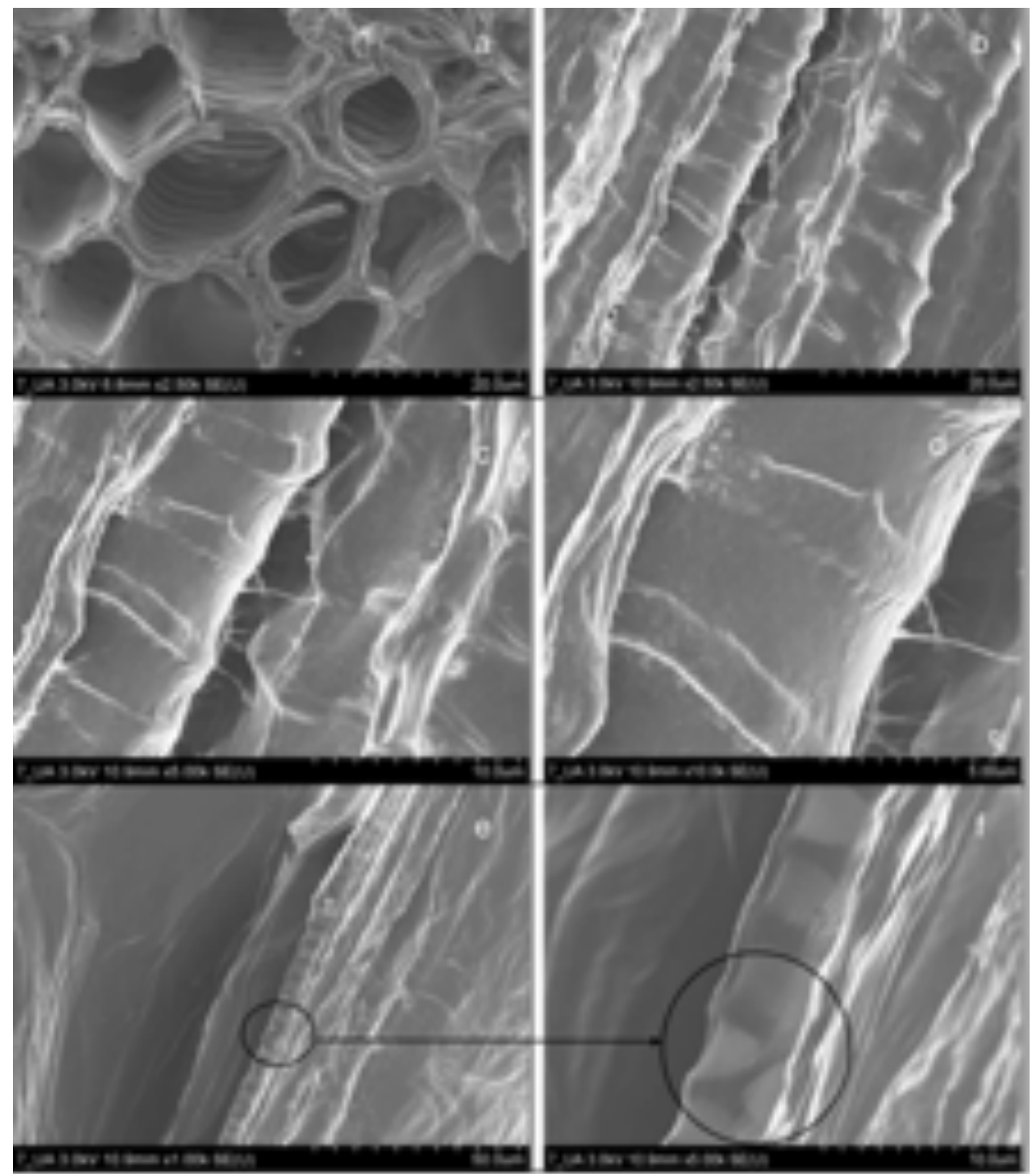

Figure 18: Spiral secondary wall thickenings in Arabidopsis thaliana

models of bifurcation and duplication. In addition, experimental confirmation of the physical processes that underpin cell division and bifurcation at multicellular scales has been demonstrated in the current paper. To this list of macroscopic quantum phenomena, we now explore the possibility of adding spin and at a theoretical level, explain how a cell, as a macroscopic quantum system, spins on its axis as it grows. 


\subsubsection{The origins of spin in a macroscopic quantum system.}

Since its discovery, spin has been considered as a physical quantity of pure quantum origin having no classical counterpart. Indeed, assuming an extension of the electron of the order of its classical radius $r_{e}=\alpha \lambda_{c}$, where $\alpha=1 / 137.036 \ldots$ is the fine structure constant and $\lambda_{c}=\hbar / m c$ is its Compton length, an angular momentum $\hbar / 2$ would involve a velocity of rotation of its surface of order $\alpha^{-1} c$, which is excluded by special relativity.

A solution to the question of the origins of spin in a macroscopic quantum system appears to present itself in earlier work $[11,32,35]$, where a new set of principles account for the origins of spin in a fractal spacetime and its relevance in standard QM, which we now briefly review.

In the first instance we consider an early model for the emergence of a spin-like internal angular momentum in fractal spiral curves [11]. The general concept, illustrated in Fig 19, shows a schematic representation of a fractal path of a particle in an intertial frame of reference. The result is a spiral path, which illustrates how "spin" naturally emerges within a 4D space-time, in which all four dimensions, including the invariant proper time $s$, are fractal. This principle has important implications in what follows.

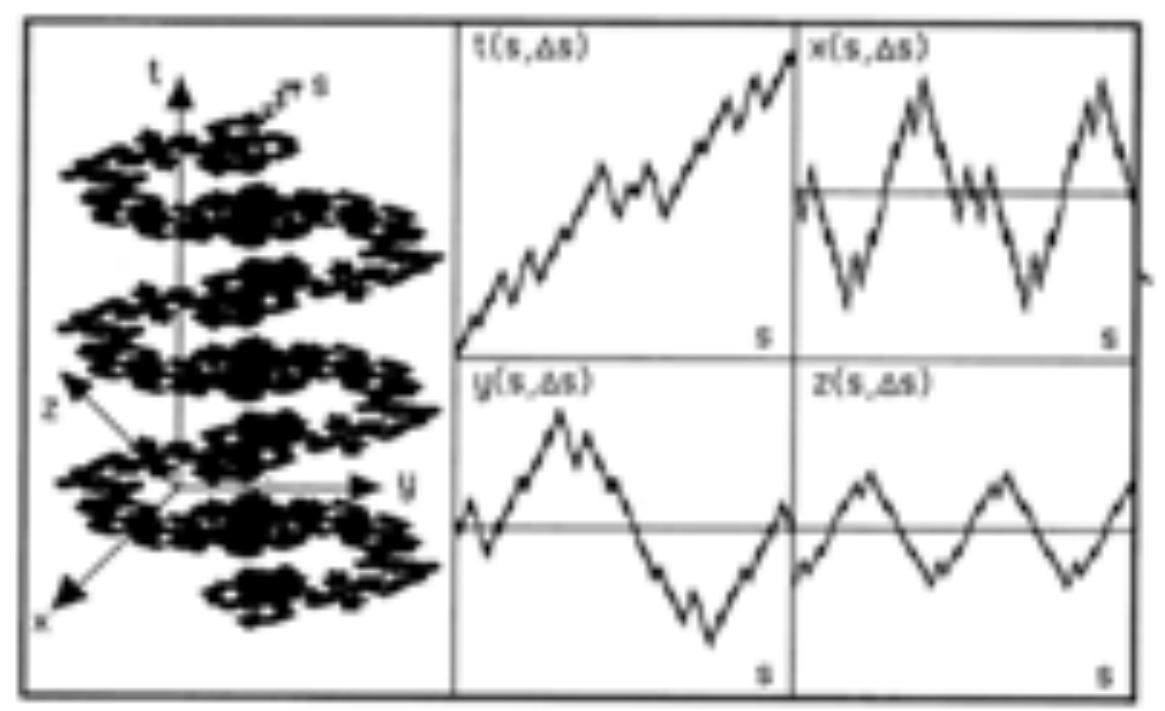

Figure 19: Schematic representation of a fractal curve in space-time from [11]. The evolution of its coordinates is described by four fractal functions of the normalized curvilinear coordinate $s$ intrinsic to the fractal curve.

As a next step we consider how a fractal space-time addresses the constraints of classical spin in a quantum system. The angular momentum $L_{z}=m r^{2} \dot{\varphi}$ of a point mass following a classical spiral path should vanish as $r \rightarrow 0$. However, in the fractal spiral model, when a scale factor $q^{-1}$ is applied to the radius $r$, the number of turns and therefore the rotation velocity $\dot{\varphi}$ is multiplied by a factor $p=q^{D_{F}}$. As a result, angular momentum is multiplied by a factor $p \times q^{-2}=q^{D_{F}-2}$, meaning it remains defined at the infinitely small limit $q^{-n} \rightarrow 0$ in the special case $D_{F}=2$. In other words, $\dot{\varphi} \rightarrow \infty$ as $r \rightarrow 0$ in such a way that the product 
$r^{2} \dot{\varphi}=0^{2} \times \infty$ remains finite when $D_{F}=2$, while it is vanishing for $D_{F}<2$ and divergent for $D_{F}>2$. This result solves the problem of the apparent impossibility to define spin in a geometric way both for an extended object and for a point-like object, and highlights the critical character of the value $D_{F}=2$ for the fractal dimension of quantum particle paths [36].

Within this context, examples of spin observed in the present work begin to make sense. For example, internal fractal geodesics of $D_{F}<2$ would explain the vanishing structures in Fig's 17a,b and d, whilst fractal geodesics of $D_{F}=2$ would account for the extended spiral structure in Fig 17f. Based on earlier work [9, 7] we make the assumption that below a critical level of charge density, we would expect a $D_{F}<2$, so we conclude that Fig's 17a,b and $\mathrm{d}$ sit within this domain.

Since $\mathrm{CK} \times 1$ leads to a charge density distribution that accommodates the range of structures we have highlighted, we would anticipate that at some point a lower [CK] would lead to a reduction in mean $D_{F}$ and in the first instance, the disappearance of the type of extended spiral structure represented in Fig 17f. By contrast, we would expect that a significantly higher $[\mathrm{CK}]$ would lead to $D_{F}>2$ and divergent spin. In experimental work to test these principles, we investigated conditions at $C K \times 0.5$ and $C K \times 4$.

After two hours at $C K \times 0.5$, cell division was at an advanced stage (Fig 20a-f) compared to $G A \times 1$, with structures being dominated by multicellular clusters. It was notable that whilst we observed chiral cells similar to Fig 17d within some of these clusters (e.g., Fig 20b), extended spiral structures reported in Fig $17 \mathrm{f}$ were absent. This outcome supports our association of the extended spiral structures with higher $[\mathrm{CK}]$ and links to fractal geodesics of $D_{F}=2$.

Following a significant increase in charge density $(C K \times 4)$, after 3 hours growth the emergence of a new class of assembly was observed (Fig 21). Whilst some examples of cell division and spin previously reported were recorded, the dominant morphology was more typical of that revealed in Fig 21c. This figure suggests that growth of $n m$-scale fibres begins with a fractal tree root-like morphology, to adhere to the surface. As the structure grows beyond a fractal base, the fibres adopt a more linear form. This pattern was mirrored in additional images in Fig's 21b and 21c, with a single, spherical cell-like structure growing from the tip. Meanwhile Fig's 21a, 21e and 21f show two or more extended linear cell structures topped with spherical cells, which appear to interact.

Note that at $C K \times 4$, a point is reached in Fig 21d where inter-fibril repulsive forces (associated with high levels of charge density) lead to the separation of individual $\mathrm{nm}$-fibrils from the main body of the stem.

The linear extended cell structures at $C K \times 4$ contrast with those at $C K \times 2$ after two hours growth, where spiral structures represent the dominant feature. We speculate that the transformation from spiral to linear structures is associated with an increase in charge packing density beyond a critical point of disorder associated with a maximum potential percolation threshold, throughout the fractal network. The physical limitations of packing beyond this critical percolation threshold, mean a move towards increased order and a decline in quantum coherence. In other words, the chaos induced symmetry breaking vanishes, with 


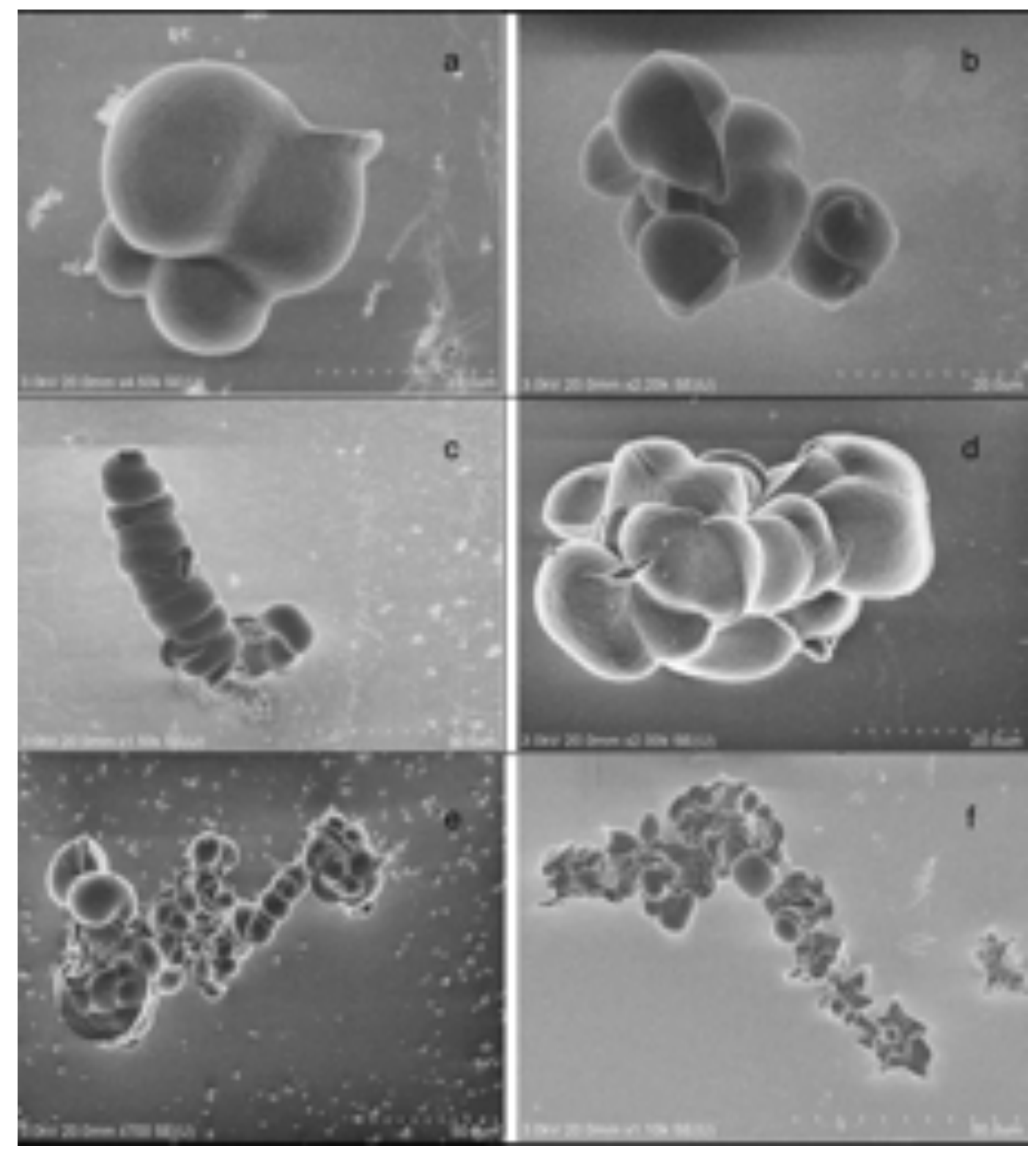

Figure 20: Examples of the growth of multicellular structures after 2 hours at CK $\times 0.5$.

symmetry being recovered at the scale at which nano-fibrils aggregate ${ }^{10}$. This is reflected in more ordered packing of dendrites in the main stem observed in Fig's 21a-f. However, we also note that in these same examples, as the initial hormone concentration is depleted during the growth process, charge density eventually declines to a point where the symmetry breaks once again and more spherical, cell-like structures emerge as secondary structures on the tips of the stems. An intriguing example of this is given in Fig 22, where a group of spiral cells emerges from an initial, linear growth at the base of the structure, as charge density

\footnotetext{
${ }^{10}$ We stress that this is not anticipated to be the case within nano-fibrils themselves, where quantum vacuum fluctuations which dominate the $n m$-scale drive their disordered internal geometry, maintaining a MQP and quantum coherence.
} 


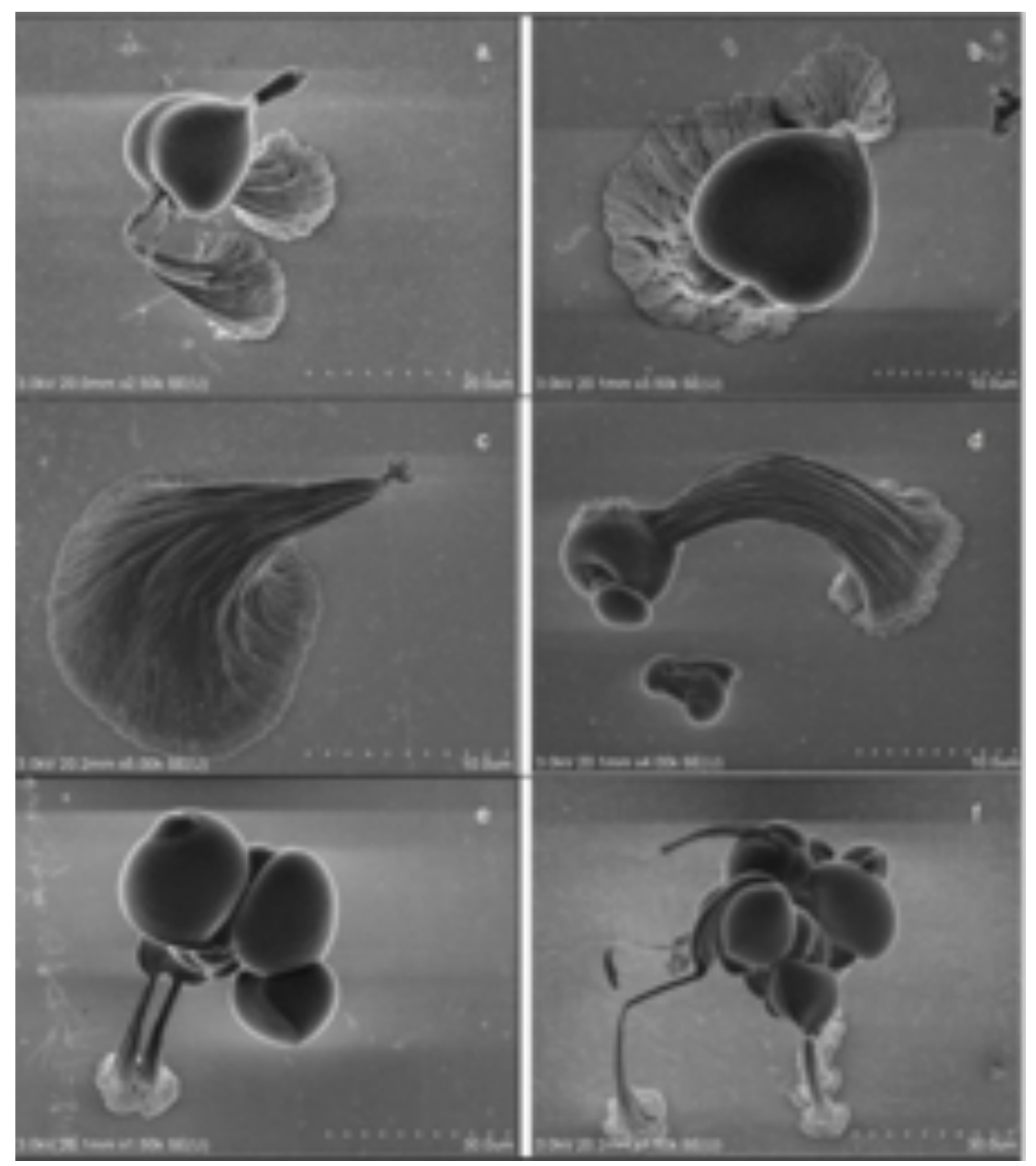

Figure 21: Examples of the growth of multicellular structures after 3 hours at $\mathrm{CK} \times 4$.

declines. The effect takes the resemblance to a gastropod beyond that of its shell. Whilst multiple shells might be discarded as impractical in an evolutionary process, the theoretical concepts developed as part of this work offer an unexpected insight into some of the physical processes at play in the emergence of such an organism.

Results of this and earlier work [7] indicate that macroscopic quantum coherence in biological systems represents just one of a more complex set of conditions shared with HTSC systems. In Fig 23 we see an adaptation of Fig 2 in [9], which represented a phase diagram for HTSC. Fig 23 represents a more generic version of the phase diagram for both HTSC and biological systems, which excludes absolute values for $\rho$ and $T$. 


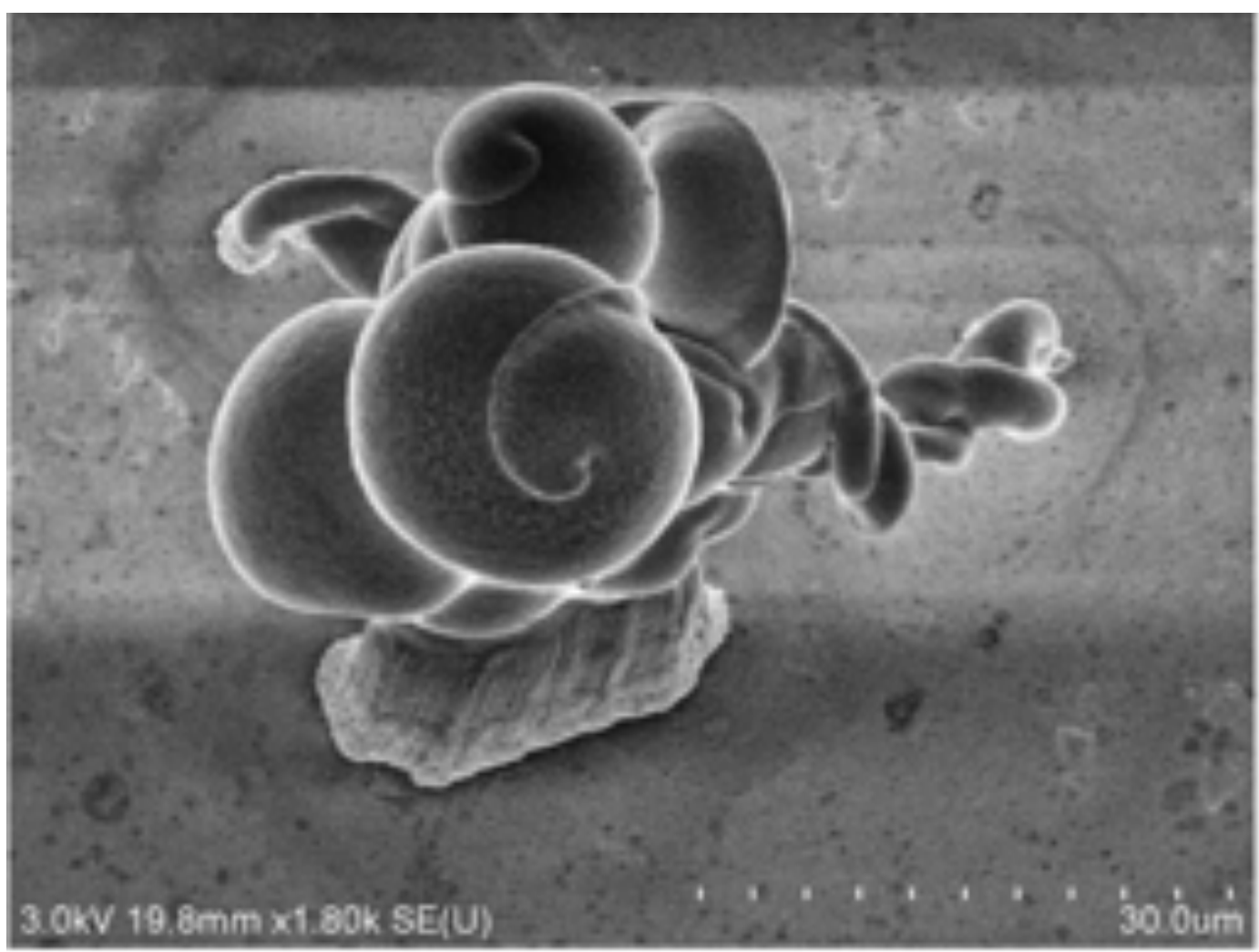

Figure 22: Example of an emergent snail-like structure after 3 hours at $\mathrm{CK} \times 4$.

Fig 23 indicates a dome of coherence associated with critical temperature $T_{c}$ in HTSC materials, with macroscopic quantum coherence being associated with a range of values for $\rho$. The peak of the dome represents maximum coherence, which correlates with a maxima for charge disorder and an associated percolation threshold for the charge induced interconnecting fractal velocity field, which underpins a MQP. We link this peak of coherence with the emergence of cell like structures, cell division and macroscopic quantum spin phenomena observed in Fig 17f. By contrast, this type of spiral phenomena has not been observed in HTSC materials due to the 2D nature of the materials that have been tested to date $[9,23]$.

A reduction in $\rho$ from the optimum associated with the peak of coherence and maximum $T_{c}$, leads to a decline in the interconnecting fractal velocity field and a corresponding decline in quantum coherence and $T_{c}$. In 3D materials reported in [7] a decline in $\rho$ is reflected in a decrease in $D_{F}$ of emergent structures. Beyond a critical point in the process of decline in $\rho$, the interconnected fractal velocity field becomes disconnected, leaving localised pools of coherence, which represent the pseudogap in Fig 23. In HTSC materials [9], localised pools of coherence within the pseudogap remain coherent at higher temperatures compared to $T_{c}$. However, due to the absence of interconnectivity associated with a critical percolation threshold, macroscopic quantum decoherence and any form of long-range order disappears, resulting in completely disordered structures. This scenario continues until $\rho$ drops to a point where a lack of repulsive charges in the system permits the formation of a lattice structure as illustrated Fig 1a. 
If we now consider an increase in $\rho$ beyond the peak of the coherence in Fig 23, packing of individual charges increases beyond the critical point of maximum disorder. This leads to an increasingly ordered packing arrangement and a decline in $T_{c}$, which we associate with a decline in the fractal velocity field and quantum coherence. At a structural level, the gradual decrease in disorder associated with an increase in packing density ultimately leads to a lattice/nematic structure as reflected in the nematic ordering of $n m$-scale fibrils in Fig 21.

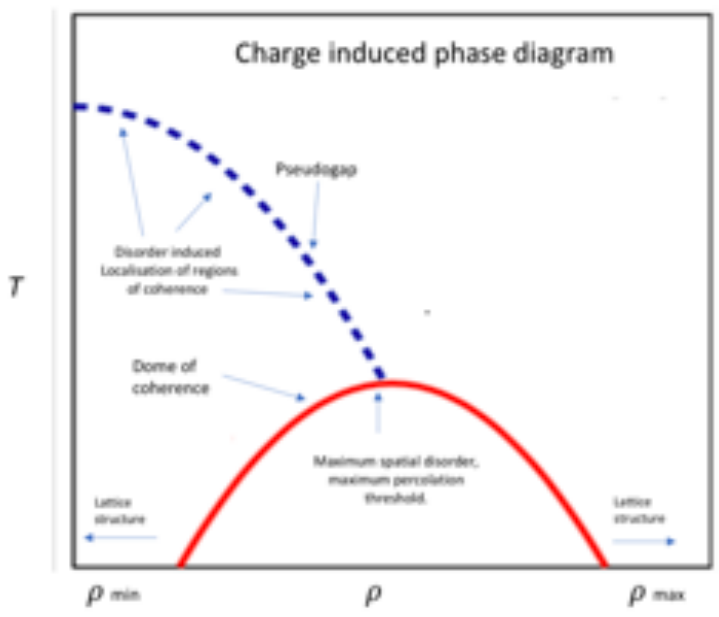

Figure 23: A generic, charge induced phase diagram for HTSC and biological systems, adapted from [9].

In a separate observation, Fig's 20d-f $(C K \times 0.5)$ illustrate cell clusters of similar size (cell count) to those observed at the higher concentration $(\mathrm{CK} \times 1)$ after 6 hours. This suggests that within the 1-2 hour time-frame, a limit is reached beyond which the growth of multicellular structures becomes constrained, without an ongoing supply of raw materials. Theory of a resource constraint is further supported by extended growth trials of up to a week in which the cell count remained comparable to that after 2 hours.

A critical resource constraint contrasts with real biological systems that are able to maintain a consistent and ongoing supply of "building materials" to sustain growth. This suggests that future work should focus on a new system design, which provides a continuous supply of fresh ingredients to support the growth of larger scale structures. It is anticipated that the outcome of such an approach, combined with the addition of a range of different charged biomolecule (e.g. hormone/protein) combinations, will lead to a diverse range of multicellular structures

On a final note in this section, having considered the role of microtubules as part of the cytosol in cell division, we now briefly consider their (separate) role in the process of cell wall formation in a plant. As discussed in Section 2.3.1, microtubules act as a "guide" for cellulose synthase complexes as they manufacture and lay down cellulose microfibrils in the process of cell wall construction. Depending on the type of cell, several different walls can be manufactured, each with a different structure and arrangement of cellulose microfibrils. 
When considering the modelling of a cell wall, each layer must be described by separate wave functions. As an example, Fig 24 represents a simple model of a cell wall in which the outcome of the underlying Schrödinger equation is governed by a separate, more narrowly defined probability density distribution.

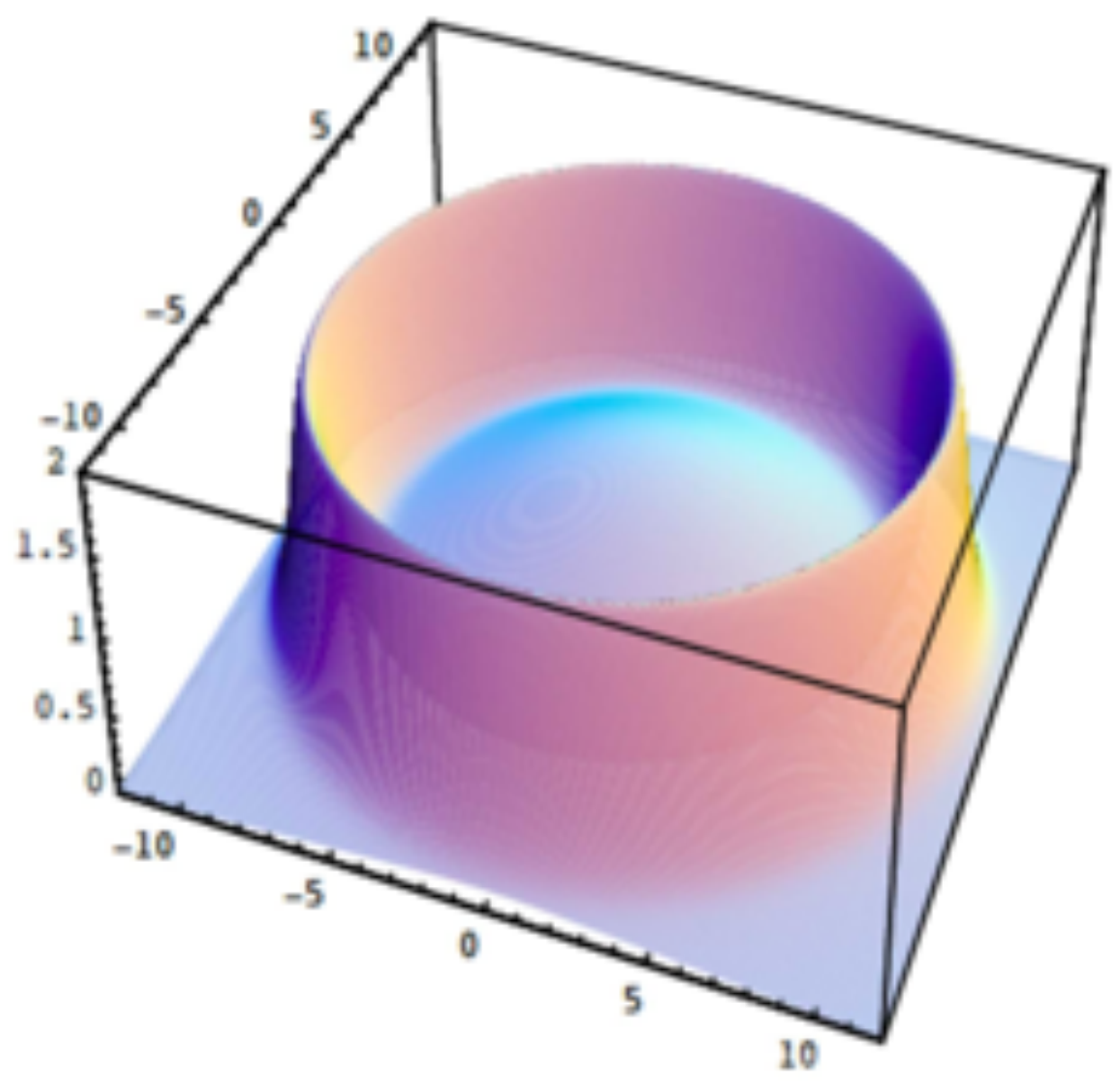

Figure 24: A 3D probability density distribution, which describes the tendency to form a cell wall structure.

In practice individual components within a cell wall, such as spiral structures illustrated in Fig 18, are considerably more complex than the representation in Fig 24. As an example, the exact translation of the spiral structure in Fig $17 \mathrm{f}$ to that of Arabidopsis thaliana in Fig $18 \mathrm{f}$ is for the moment unclear. Whilst Fig 16b in [7] confirms the fractal $n m$-scale architecture that underpins this type of structure, studies reported in [24] confirm their deposition is not directly associated with the cytosol. A separate, transitory network of microtubules and Microtubule Associated Proteins (MAP's) guides the deposition of cellulose microfibrils within the cell wall.

In a second example, we note that the nematic assembly of $\mathrm{nm}$-scale dendrites in this work at $C K \times 4$ closely relates to the arrangement of cellulose nano fibres within structural plant 
cell walls, where nano fibres can be oriented parallel with the main axis of the cell [37]. Fig's 9 and 10 in [37] represent examples of a nematic arrangement of cellulose nano-fibres within a cell wall. Discussion of this result in [7] suggested an association with very low levels of charge density. Whilst this is not ruled out by these latest findings, we can now propose a more probable association with high rather than low levels of charge density. Meanwhile, at a larger scale we note that a nematic arrangement of nano fibres found within an individual cell is mirrored at the scale of the whole tree, where structural cells generally align parallel to the stem of the plant.

\subsubsection{A proposed mathematical framework to accommodate spin within a macroscopic quantum system.}

\subsubsection{Derivation of the Pauli equation.}

Having considered the principles that underpin the origins of spin in a fractal spacetime as a geometric principle in Section 2.3.3, we now consider how it fits within the framework of QM.

Intrinsic spin is conventionally described by the Dirac equation (for relativistic QM) and the Pauli equation at its non relativistic limit [8, 11,35]. Given the diffusion limited rate of cell growth, which leads to the emergence of spiral structures, the Pauli equation therefore offers the most appropriate framework for an initial consideration.

In order to account for what we have observed in terms of the Pauli equation, we briefly review the physical principles which underpin it. As a first step in order to maintain consistency between relativistic and non relativistic scenarios, we substitute $t$ with the invariant proper time $s$. However, as we shall see, the velocity field and therefore the wave function are not explicit functions of $s$. Within this context, we recall that within the scale relativity framework, the origin of the complex wavefunction and the Schrödinger equation is underpinned by breaking of the symmetry $d s \leftrightarrow-d s$, which leads to a two valuedness, reflected in two total derivatives $d_{+} / d s, d_{-} / d s$. Meanwhile, the inclusion of spin comes from a discrete symmetry breaking on the space-time differential element $d x^{\mu} \leftrightarrow-d x^{\mu}$. This emerges from the fact that in general, $v\left[x^{\mu}\left(s, d x^{\mu}\right), s\right] \neq v\left[x^{\mu}\left(s,-d x^{\mu}\right), s\right]$, where the velocity field $v$ is a function of the fractal coordinates, which in turn depend on the four resolutions $d x^{\mu}$, with $\mu=0,1,2,3$.

This second two valuedness, accounted for through algebra doubling from complex numbers $\mathbb{C}$ to quaternions $\mathbb{H}$, corresponds to a second doubling of the total derivatives to give $d_{++} / d s, d_{+-} / d s, d_{-+} / d s$ and $d_{--} / d s$, and a doubling of the partial derivative $\partial / \partial x^{\mu} \rightarrow$ $\left(\partial_{+} / \partial x^{\mu}, \partial_{-} / \partial x^{\mu}\right)$.

Because the quaternion algebra is isomorphic to the rotation group algebra $S O(3)$, itself related to $S U(2)$ by homomorphism, we can consider the two one-dimensional irreducible representations of $S U(2)$ over the quaternions. One is the trivial spin-0 representation. The other is the spin-1/2 representation, which acts on a one-component quaternion basis $\phi$. 
Instead of writing the quaternion as a function of its four components: $\phi=\phi_{0}+i \phi_{1}+$ $j \phi_{2}+k \phi_{3}$, we can write it as a function of its symplectic components. These are elements of a complex subspace of the quaternionic algebra spanned by 1 and $i$, denoted $\mathbb{C}(1, i)$. In the symplectic representation $\phi=\phi_{\alpha}+j \phi_{\beta}$, the symplectic components are defined as $\phi_{\alpha}=\phi_{0}+i \phi_{1}$ and $\phi_{\beta}=\phi_{2}-i \phi_{3}$.

The induced action of the generators of the spin- $1 / 2$ representation of $S U(2)$ on the twocomponent column vector

$$
\Phi=\left(\begin{array}{c}
\phi_{\alpha} \\
\phi_{\beta}
\end{array}\right)
$$

is given by the $2 \times 2$ Pauli spin matrices. This leads to a doublet $\left(\psi^{1}, \psi^{2}\right)$ of complex wave functions (a Pauli spinor), which naturally emerges within the framework of manifolds carrying a quaternionic structure [38].

On first consideration, one might expect that the Pauli equation should logically proceed from the symmetry breaking $d t \leftrightarrow-d t$ (for a non-relativistic motion) and $d x^{\mu} \leftrightarrow-d x^{\mu}$ (for the appearence of spinors). However, as shown by Célérier and Nottale [35], it is not possible to use this approach to directly write a non-relativistic equation for spin- $1 / 2$ particles. It can only be derived as a non-relativistic approximation of the Dirac equation. This requires a further (third) doubling linked to breaking of the symmetry $x^{\mu} \leftrightarrow-x^{\mu}$, due to the breaking of parity $P$ and time-reversal $T$ symmetries specific to the relativistic case. This third doubling means the four total derivatives double once again to become $\tilde{d}_{++} / d s, \tilde{d}_{+-} / d s, \tilde{d}_{-+} / d s$ and $\tilde{d}_{--} / d s$.

For each of these cases, one finds a total derivative

$$
\frac{d}{d s} \pm \pm=\frac{\partial}{\partial s}+v_{ \pm \pm}^{\mu} \partial_{\mu} \mp \frac{\lambda}{2} \partial^{\mu} \partial_{\mu}
$$

and its tilde counterpart. These transformations are accounted for through the introduction of a bi-quaternionic velocity field, composed of eight velocity fields $\left\{v_{++}^{\mu}, v_{+-}^{\mu}, v_{-+}^{\mu}, v_{--}^{\mu}\right\}$ and $\left\{\tilde{v}_{++}^{\mu}, \tilde{v}_{+-}^{\mu}, \tilde{v}_{-+}^{\mu}, \tilde{v}_{--}^{\mu}\right\}$. For a detailed mathematical description of the bi-quaternionic velocity field (Eq 24) and subsequent steps, which lead to a derivation of the Pauli Equation see Section 5 (supplementary material).

\subsubsection{Numerical simulation of spin.}

Having described key elements that underpin the Pauli equation in Sections 2.3.4.1 and 5 (Supplementary material), we next consider earlier work [35], which confirms the physical picture of spin emerging from a fractal geometry based on explicit solutions of the Pauli (or Dirac) equation.

We begin by decomposing elementary displacements in a fractal space-time into classical (differentiable) and fractal (nondifferentiable) components. 


$$
d X_{ \pm \pm}=v_{ \pm \pm} d t+d \xi_{ \pm \pm}
$$

Geometric fractal fluctuations are replaced by a stochastic variable such that $\left\langle d \xi^{2}\right\rangle / c d t=\lambda_{c}$ and $\langle d \xi\rangle=0$. Then after the velocity fields have been recombined in a unique biquaternionic form they become a solution of the bispinorial geodesic equation Eq.31, which can be integrated in terms of the Dirac equation, whose non-relativistic limit is finally the Pauli equation.

Solving the Pauli equation for a given physical problem yields a quaternionic wave function

$$
\psi=\eta_{0}+i \kappa_{0}+\left(\eta_{1}+i \kappa_{1}\right) e_{1},
$$

from which the velocity fields can be derived

$$
\begin{array}{r}
v_{++}^{\mu}=-\frac{\mathcal{S}_{0}}{m c}\left(\eta_{0} \partial^{\mu} \kappa_{0}+\kappa_{0} \partial^{\mu} \eta_{0}+\eta_{1} \partial^{\mu} \kappa_{1}+\kappa_{1} \partial^{\mu} \eta_{1}+\right. \\
\left.+\eta_{0} \partial^{\mu} \eta_{0}-\kappa_{0} \partial^{\mu} \kappa_{0}+\eta_{1} \partial^{\mu} \eta_{1}-\kappa_{1} \partial^{\mu} \kappa_{1}\right) .
\end{array}
$$

One can then construct various realizations of the geodesics by performing numerical integrations of the stochastic differential equation

$$
d X_{++}=v_{++} d t+\zeta \sqrt{\lambda c d t}
$$

in which the explicit form of $v_{++}$, given by Eq. (18), is inserted, and where $\zeta$ is a normalized stochastic variable such that $\left\langle\zeta^{2}\right\rangle=1$ and $\langle\zeta\rangle=0$.

Adopting the following spinor wave function from Dezael [39],

$$
\psi=A_{0} e^{-\frac{i}{\hbar}\left(\vec{p} \cdot \vec{r}-E_{0} t+\sigma_{0} \phi\right)}+A_{1} e^{-\frac{i}{\hbar}\left(\overrightarrow{p_{1}} \cdot \vec{r}-E_{1} t+\sigma_{1} \phi\right)},
$$

the biquaternionic velocity field, given by $m \vec{V} \simeq i \hbar \psi^{-1} \vec{\nabla} \psi$ can be derived, which must be such that $\vec{v}_{-+}=-\overrightarrow{\vec{v}}++$ in the nonrelativistic approximation considered here. This is possible only provided $\overrightarrow{p_{0}}=\overrightarrow{p_{1}}$, and $\sigma_{0}=\sigma_{1}[39]$. These conditions simplify the expression of the biquaternionic velocity, which is reduced to

$$
\vec{V} \simeq \vec{v}_{++} \simeq \frac{1}{m}\left(\vec{p}_{0}+\frac{\sigma_{0}}{r \sin \theta} \overrightarrow{u_{\phi}}\right)
$$

where $\overrightarrow{u_{\phi}}$ is the unitary vector associated to the rotation by $\phi$ around $\overrightarrow{p_{0}}$. Dezael finally obtains the following differential equations, now written in Cartesian coordinates :

$$
\left\{\begin{array}{c}
\dot{x}=-\frac{\sigma_{0}}{m} \frac{y}{x^{2}+y^{2}} \\
\dot{y}=\frac{\sigma_{0}}{m} \frac{x}{x^{2}+y^{2}} \\
\dot{z}=\frac{p_{0}}{m} .
\end{array}\right.
$$


These equations clearly describe a class of spiral motions such that $m r^{2} \dot{\varphi}=\sigma_{0}$, where $r$ can take any value, so that $r \rightarrow 0$ implies $\dot{\varphi} \rightarrow \infty$.

Then one may finally carry over this expression for the classical velocity field into the stochastic differential equation (Eq 19) and integrate it numerically. An example of one of the spiral fractal paths obtained in this process is shown in Fig. 25, which reveals spin as an emergent property of $4 \mathrm{D}$ fractal space-time. This exact geometric description, whose spin is quantized in units of $\hbar / 2$, supports the main features of the earlier rough fractal model in Fig 19.

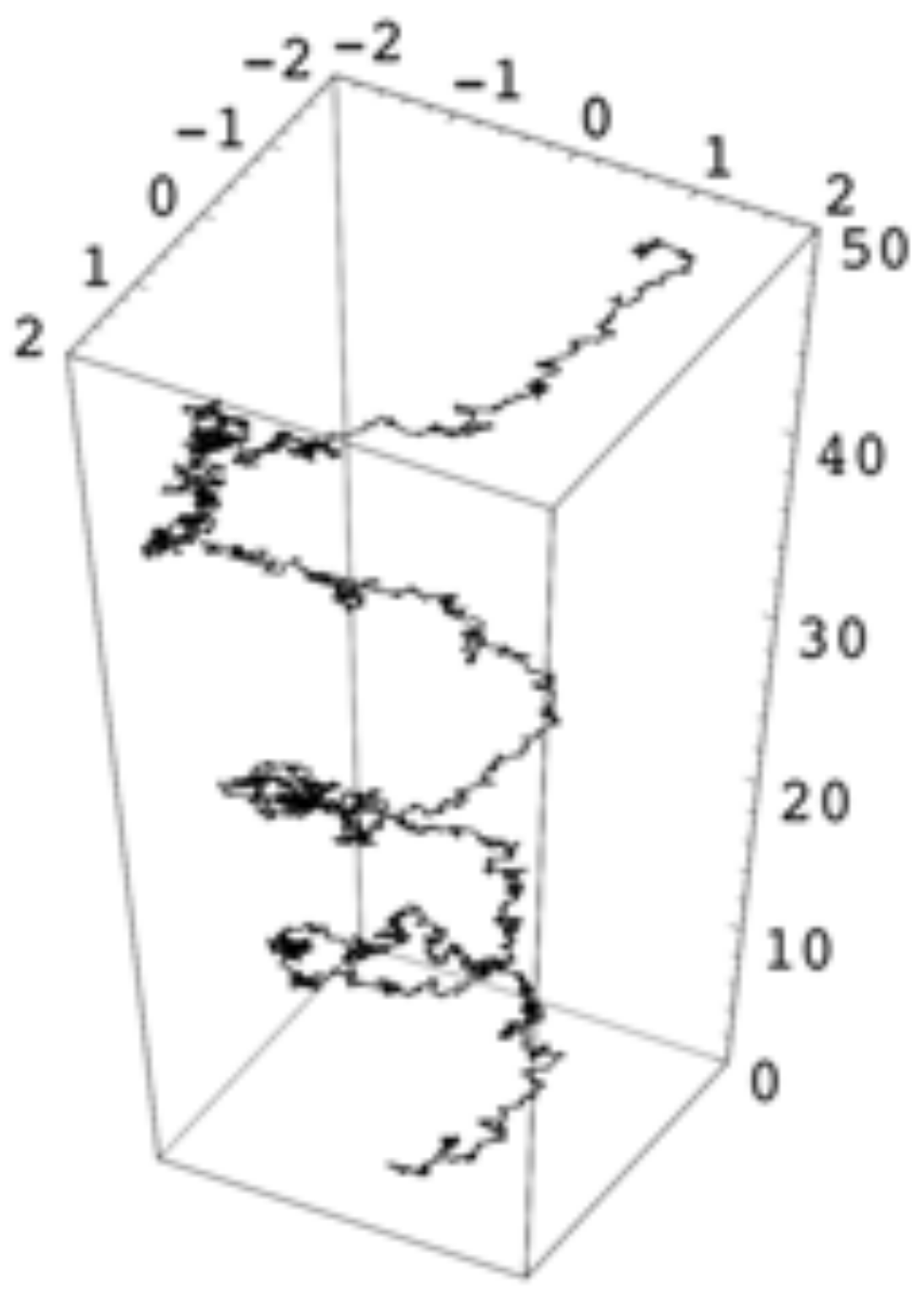

Figure 25: Numerical simulation of a typical spinorial geodesic in a fractal space, from [35]. The plotted curve corresponds to the following values of the parameters: $\widetilde{D}=\hbar / 2=0.05, d t=0.01$, $\sigma=1 / 2, v=1$. A large value was chosen for the spin, $\sigma=5 \hbar$, to fully reveal the spiral trajectory. 


\subsubsection{Equating spinorial geodesics with experimental observations.}

The spiral in Fig. 25 represents just one of an infinite number of possible realisations of an electron as a point particle. This contrasts with the macroscopic spiral in Fig 26, which reflects the physical integration of a consecutive series of slices of a 2D fractal space, analogous with Fig 19. The structure that emerges from integration of this set of fractal geodesics dictates that time must now also be fractal, creating a composite 4 dimensional spacetime construct, which underpins a macroscopic quaternionic wavefunction (Pauli spinor). The result illustrates the fundamental relativistic and quantum nature of spin (even in a non-relativistic scenario), which is attributed to the non differentiability of the quantum space-time geometry and not only of the quantum space [8].

A further difference between Fig 26 and a set of electron trajectories is that only the field (defined by a MQP), is coherent rather than a fully coherent fluid, vortex-like structure in the electron case. The macroscopic spiral structure in Fig 26 represents an emergent matter wave (rather than a point particle), which rotates in a fractal 4D spacetime as a continuous wavefront, at non-relativistic speeds.

One of the key challenges in standard QM lies in the most appropriate way to visualise a particle such as an electron as a matter wave. In Fig 26 we speculate that we may have a macroscopic realisation of the concept. Within this context, it seems plausible to consider a particle such as an electron in an analogous manner, as a fluid of geodesics rotating in a superfluid quantum vacuum. However, since the matter wave associated with an electron is a completely coherent, quantized packet of matter it would have to form a recirculating (quantized) vortex, or toroid ${ }^{11}$. It's clear that this description does not apply to Fig 26, which reflects continuous growth, rather than a recirculating, localised, fixed unit of matter.

Adopting the model of a quantum particle as a recirculating vortex offers an interesting, alternative perspective on the "two-cell" double helix model proposed for Fig 26 in Section 2.3.2. We speculate that with the emergence of a synthetic cell with the critical internal fractal structure $D_{F}=2$, macroscopic quantum forces are sufficient to induce a rotational action and the emergence of a vortex-like structure. As the cell grows in length, rotational forces will lead to the emergence of a natural spiral structure such as Fig 26. Under these conditions, the suppressed centre of the vortex creates the appearance of a double helix. In other words, instead of a travelling, rotating wave front for a quantum particle such as an electron, we observe the evolution of the wave front as an aggregated time series, with not just one, but all probable outcomes (paths) being revealed, along with its wavelength $\lambda \approx 6 \mu \mathrm{m}$ (in Fig 26), which is close to that in Fig $18 \mathrm{f}$ at $\lambda \approx 5 \mu \mathrm{m}$. This puts both examples in the mid infrared range of 3-8 $\mu m(37-100 \mathrm{THz})$.

In the case where charge density in the form of [CK] begins to decline, we see an associated decline in $D_{F}\left(D_{F} \rightarrow<2\right)$ and a reduction in energy in the system, with the continuous helix replaced by a larger diameter, vanishing spiral, as observed in Fig 27.

\footnotetext{
${ }^{11}$ Under this interpretation the toroidal nature of the electron precludes its existence as a point particle and offers a different perspective on the elimination of infinities traditionally associated with a point particle, highlighted in Section 2.3.3. Discussions on the full details and implications of this principle fall outside the scope of the present study and will be dealt with in a forthcoming paper.
} 


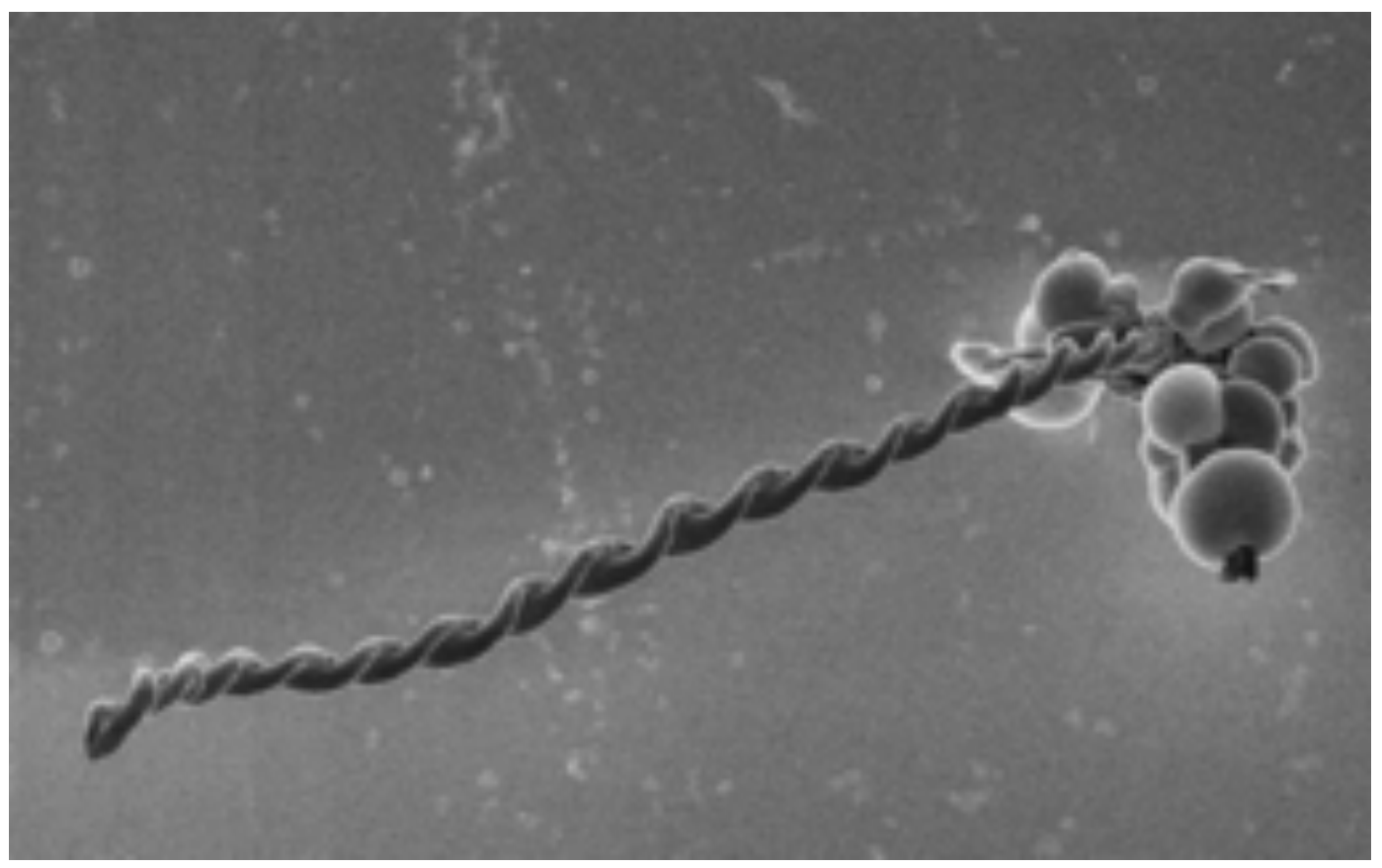

Figure 26: A left-handed helix emerging as a result of a traveling rotating wave package, with each stage of assembly adding to previous stages, to create a continuous, aggregated time series.

It is not possible to replicate all aspects of quantum spin in standard QM. However, within this new macroscopic framework, we appear to have confirmed earlier predictions [35], which suggested the possibility of an experimental construction of fractal spiral trajectories that leads to the emergence of key properties associated with quantum spin, which are recovered as an approximation.

Confirmation of the critical nature of fractal space-time in the emergence of intrinsic spin highlights the limitation of the Schrödinger equation in both standard and macroscopic QM. As a first approximation we therefore propose a macroscopic form of the Pauli equation to represent the broader set of phenomena (now including spin), that we now associate with macroscopic QM. Adopting this approach, we treat the Pauli equation (Eq 53) in the same way as the Schrödinger equation in its more generic form (Eq 10), where $\hbar=2 m \widetilde{D}$ in standard QM. The result (Eq 23) is a new, more generic version of the Pauli equation

$$
i \widetilde{D} \frac{\partial}{\partial t}|\psi\rangle=\left[\frac{1}{2 m}(i \widetilde{D} \nabla-q \mathbf{A})^{2}-q \widetilde{D} \sigma \cdot \mathbf{B}+q A_{0}\right]|\psi\rangle
$$

in which the Pauli Spinor

$$
\phi=\left(\begin{array}{c}
\psi_{+} \\
\psi_{-}
\end{array}\right)=|\psi\rangle,
$$

and charge $(e)$ is replaced with more generic notation $q$, which could potentially include 


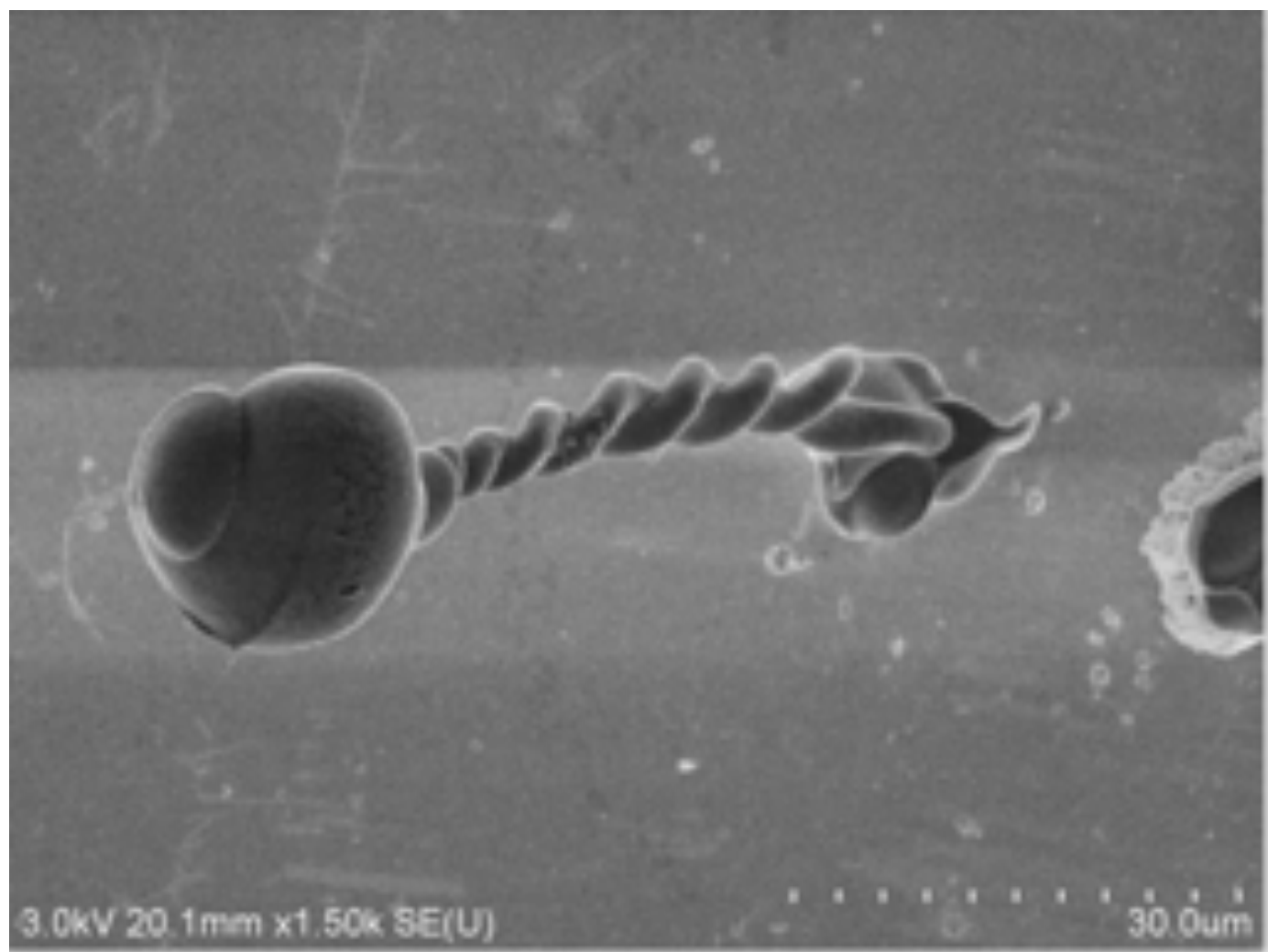

Figure 27: An example of the transition of a continuous rotating structure, where $D_{F}=2$, to a vanishing spiral as charge density declines and $D_{F} \rightarrow<2$.

mean charge on a biomolecule or macroscopic quantum object such as a protein complex or a cell.

Substitution of the macroscopic Schrödinger equation with a new, macroscopic Pauli equation (Eq 23), allows the inclusion of intrinsic spin alongside the already extensive list of macroscopic quantum phenomena reported in this and earlier studies [7].

\subsubsection{General discussion.}

One of the challenges to acceptance of the proposal of macroscopic quantum systems playing a key role in biological systems lies in the concept that biological systems represent open systems, in constant flux with the environment. By contrast a quantum system is seen as closed, being described by an equation of state such as a Schrödinger equation (Eq 4). However, in reality, a Schrödinger equation describes an internal system in constant interaction with its environment, represented at a generic level by an external potential $(\phi)$. The quantized system is maintained as long as internal quantum forces exceed external diffusive forces. When external forces exceed internal forces, the quantum system collapses to its pointer states, leading to decoherence [7].

A key difference between this and a biological system is that the latter grows over time 
as a system in dynamic interaction with its environment. The system is dependent upon various inputs such as energy, nutrients, oxygen, carbon dioxide and ions (which dictate charge density), which represent a key element in the emergence of structure, to support its maintenance and growth. However, as we have shown in this and earlier work [5, 6, 7], the system grows in a quantised way, constructed through a complex set of multi-scale assembly processes, with each macroscopic wave function being generated from the square root of charge density $(\psi \propto \sqrt{\rho})$, with each unit of charge being associated with a separate wave function at a lower scale.

At the smallest scale of interest within the present context, individual ions, nucleic acids, amino acids and hormones have the potential to play a key role in the emergence of structures such as proteins and $\mathrm{nm}$-scale cell constituents. As macroscopic quantum objects, these $\mathrm{nm}$ scale structures then assemble to create a broad range of larger, sub-cellular structures, which then in turn assemble further to form a large and diverse range of cells. Finally, at the level of a living organism, individual cells act as macroscopic units of charge in a multicellular network, which has the potential to create an interconnected, multi-scale system of MQP's, which form the basis of a macroscopic wave function and a macroscopic equation of state, such as a Schrödinger or Pauli-like equation. Despite the extra complexity described above, at one level we see an analogy with a process in standard QM such as molecular assembly to create an amino acid. However, such an analogy reflects only a fraction of the scenarios that we have considered to date. As highlighted in Fig 23, a range of conditions lead to different patterns of long-range order and structure, which reflect a range of values for coherence and decoherence.

\section{Conclusions}

The principle objective of experimental work was to improve our understanding of the detailed physical processes that underpin cell division. Results indicate that under specific conditions, a solution of $\mathrm{BaCO}_{3}-\mathrm{SiO}_{2}$, plus GA or CK leads to the growth of a high density fractal network of dendrites (creating a macroscopic path integral), which takes the form of a synthetic cell-like structure with the intrinsic capability to divide. As an approximation, this structure appears to be analogous with the fractal network of proteins (microtubule and actin filaments) that constitute a cell's cytoplasm, or more specifically the cytosol.

Results indicate that the cytosol, as an integral part of a living cell, underpins a chargeinduced macroscopic quantum system, which supports theory of the emergence of a cell and its subsequent division as macroscopic quantum processes.

At another level, the results support theory in [7] that the genome and its interaction with the environment, expressed through the transcriptome, proteome, metabolome, fluxome and electrome, dictates a range of physical conditions, which lead to the emergence of the many different structures and processes that we associate with living systems.

By decomposing complex systems such as cell formation and division into more basic processes, we have demonstrated the principle that it is possible to describe such a system with an equation (or set of equations) of state, such as a Schrödinger or Pauli equation. 
Bifurcation processes, driven by environmental (quantum vacuum/thermodynamic) fluctuations (illustrated in Fig 2) are fundamental to the emergence of macroscopic path integrals, which underpin macroscopic quantum systems (order out of chaos). Depending on conditions, the process of bifurcation repeats itself over a broad range of scales, to create a diverse range of structures, from proteins to cell organelles and cell walls, which constitute a living cell. The cell as the 'quanta of life', then has the potential to repeat the process, building new networks and structures through spontaneous duplication and bifurcation to create multicellular organisms.

Whilst the overall set of processes involved in real cell division are undoubtedly more complex than those described in the present synthetic approach, it is revealing to see that a cell division-like process can be induced by a single hormone such as GA or CK as part of a synthetic hydrogel-like cytosol. The results therefore provide an effective demonstration of the fundamental physical nature of a real cell division process, in which the cytosol represents the key ingredient, with disorder induced macroscopic quantum coherence being fundamental to the duplication process. Without it the replication of genetic material and the emergence/evolution of the vast range of structures and functions within more complex multicellular organisms could not exist.

These basic principles do not detract from the very complex set of synchronised processes operating within a living cell that still require more careful analysis. At one level, this work sheds new light on the role of multi-scale fractal structures and their ubiquity in biological systems, with the emergence of larger scale ordered structures being governed by competition between the external diffusive force, and internal macroscopic quantum forces. At another level genetic diversification has led to almost infinite set of potential conditions, principally defined by charge density (ion and charged biomolecule concentration) and its distribution, which leads to a vast range of possible structures.

As noted previously [6], not all properties associated with standard quantum mechanics are recovered in the macroscopic theory. The application of the scale relativity/fractal spacetime theory to standard QM is based on the description of space-time, toward small scales, as a pure, fully non-differentiable continuum. Within this framework, "particles" (accounted for by a wave function) reflect the various manifestations, of the geodesics of this nondifferentiable continuum in which scale divergence is without any lower limit, so that there is no hidden parameter in this case. The various properties of elementary "particles" (mass, charge, spin, etc.) emerge as manifestations of the geometric properties of this fluid of geodesics. There is no "trajectory", but only purely geometric paths. Quantum phenomena such as entanglement, indistinguishability of identical particles, or spin-statistics relations are simple and natural consequence of such a purely geometric description.

Within the context of macroscopic quantum-type systems, based on a macroscopic constant larger than $\hbar$, the situation is different and therefore also the interpretation. These systems are characterized by their fractality on a given range of scales, with an upper limit (the Einstein-de Broglie scale) as in standard quantum mechanics, but now also a lower limit. In this case there are particles in the common sense which follow trajectories, even though their possible realizations are infinite in number, fractal and locally irreversible, which leads to quantum-type equations. 
The two situations therefore share a description in terms of wave function solutions of a Schrödinger equation (although not with the same constant). They therefore share most of the consequences of this behaviour, except those derived from complete non-differentiability (entanglement, indistinguishability, etc.). In practice, this means that the type of macroscopic quantum systems considered in the current work differ from standard QM in that only bosons remain coherent, leading to a new class of quantum system [7, 9, 23]. This is exemplified in proteins $[15,16]$, photosynthetic systems [29, 30], High Temperature Superconducting materials [9, 19, 20, 21, 23] and Coherent Random Lasing [9, 28].

Whilst the systems considered in the present work are only partially coherent, many phenomena associated with standard quantum theory are recovered, including quantization, nondissipation, self-organization, confinement, structuration conditioned by the environment and macroscopic quantum decoherence. In addition, we now have experimental evidence to support the principle of evolutionary time described by the time dependent Schrödinger equation, which describes models of bifurcation and duplication.

Finally we also have evidence of intrinsic spin in macroscopic quantum systems, which can lead to disorder-induced spiral structures when charge density and its spatial distribution meets critical criteria. The results support earlier theory [11, 35, 40] of intrinsic spin as a pure quantum process, that naturally emerges from a 4D fractal space-time, which can be accommodated within the framework of the Pauli equation, as a non-relativistic approximation of the Dirac equation.

As implied from the discussions above, there is a difference between macroscopic and "standard" quantum spin, which corresponds to $m r^{2} \dot{\varphi}$, where $r \rightarrow 0$ and $d t \rightarrow 0$, without any lower limit. In a macroscopic scenario the range of scales of the fractal helix structures which underpin the apparently intrinsic angular momentum (i.e. spin) may be large, but there is always a definable lower limit.

This scenario appears to be very similar to the turbulence case [41] where the divergence of acceleration with scale is huge (reaching thousands of $\mathrm{g}$ in simple von Karman experiments at a scale of $20 \mathrm{~cm}$ ), but stops at the Kolmogorov dissipation/viscous scale.

The central principle of macroscopic quantum coherence established in this and earlier work such as $[7,8,9,23]$ is supported at a more generic level by Geesink and Meijer [42, 43] who collated electromagnetic frequency data from a large number of different biological studies. After normalisation, the data sets were found to aggregate around 12 preferred frequencies, creating a "musical tonal scale". Geesink and Meijer attribute these preferred frequencies to "pointer states", which we consider in Section 1.3 with respect to collapse of the fractal velocity field to its more stable roots during quantum decoherence.

At a different level, the set of frequencies reported in [42, 43] were considered within the framework of a rotating vortex or torus as a vehicle for quantum wave transition. Our theoretical and experimental work supports this hypothesis within certain limits. However, the key principle established in the present paper indicates that macroscopic quantum coherence is underpinned by a broader based quantum field theory, within which the emergence of a vortex-like, rotating structure represents just one outcome, under a specific set of conditions. 
We stress the point that this new quantum field theory has yet to be described in a comprehensive manner in either macroscopic or standard QM. Within this context, the proposal of a macroscopic Schrödinger or Pauli equation represents only a first approximation. As in standard QM, these equations do not help our understanding of the detailed mechanism that underpins quantum decoherence. Dealing with this issue requires a significant new step in our understanding of the foundations of quantum mechanics, which requires a detailed mathematical description. Such a challenge falls outside the remit of the current study and will be reported in a future paper.

Considering the broader implications of the present work, results offer a number of important new insights into the emergence of life. Given an appropriate set of initial conditions, a putative cell in the form of a dendritic fractal cytoplasm could have emerged as a crude "celllike" macroscopic quantum object, with the intrinsic capacity to divide. If a metaphorical protein soup in a primeval swamp was able to support an ongoing supply of ions, amino acids and proteins, it could have signalled the spontaneous emergence and evolution of both single and multicellular structures. Under such conditions, DNA as we know it would not have been an essential ingredient in the early stages of such a process, but could have started its evolutionary process in parallel with other structural developmental processes. However, whilst DNA may not have been an essential requirement of early, putative life forms, it would have been essential for later development, creating the proteins and metabolites required to support macroscopic quantum processes in the absence of an initial set of start up conditions.

This hypothesis sits well with a proposal by Melkikh and Meijer [44], that previously unconsidered long-range forces (which we have explicitly linked to the quantum vacuum and disorder-induced MQP's), could play an important role in the evolution of the complex folding processes associated with DNA and protein structures. A detailed discussion on this particular topic falls outside of the scope of the present paper. However, the new approach to self organization established in this and earlier work [7] offers a new framework within which to study mesoscale processes such as protein folding from first principles.

Bearing in mind the relative simplicity of the processes outlined in the emergence of multicellular structures, it raises questions about why the eukaryotes took so long to evolve. The answer almost certainly lies in the time required for the evolution of the vast range of additional structures (e.g organelles) and dynamic, interdependent processes associated with a biological system.

The results so far provide convincing evidence that biological processes, structures and systems are in no way privileged. The mystique associated with living systems is tied up in the vast set of complex, interacting systems, which goes some way to accounting for the challenges associated with the deconstruction of processes into their individual component mechanisms. However, as a first step in understanding this complexity, we have shown that it is possible to recover differentiability and reversibility at the scale of macroscopic wave functions which define structures and processes at different scales.

This brings us back to the question posed by Schrödinger [45]; "What is life"?

We now have a growing body of evidence to support theory that macroscopic quantum processes play a key role in living systems, which adds a new dimension to the concept of the 
"electrome" introduced by Loof [46]. If this theory is correct, then examples of macroscopic quantum processes discovered in the field of quantum biology to date represent the tip of the iceberg. Indeed, macroscopic quantum processes may prove to become a defining criteria of what constitutes a living system.

\section{Future work}

The results of this and earlier work [5, 6, 7] represent an important base upon which to establish a longer term research and development program that continues to build on our understanding of the detailed physical processes that underpin biological systems.

In [7] we gave just a few examples of possible emergent structures using $\mathrm{CO}_{2}$ as the source of charge density. In the work presented here, we have demonstrated the potential to mimic biological systems through the use of charged biomolecules to more precisely control the emergence of cell-like structures, cell division and the growth of simple, multicellular structures. However, within the present experimental system, the growth of larger and more complex structures is constrained by a consumption led decline in key ingredients over time.

A future challenge lies in the development of a system that mimics a biological system more rigorously. As a first step this would involve the design of a system, with a feedback loop, which provides a continuous supply of material and a constant set of conditions (over time), to support the growth of large-scale, multicellular structures. Managing this process is particularly relevant when considering the sensitivity of these emergent processes to varying conditions. As part of such a process it will be important to develop appropriate techniques to monitor the growth of these new structures in real time. The use of environmental electron microscopy is one possible approach which needs to be evaluated. However, it is possible that the electron beam could lead to decoherence of the structuring field. One proposed alternative approach includes the use of live cell imaging techniques using TimeLapse microscopy reported in [24].

A successful system design should support cell formation and duplication processes, which can lead to a new, "cellular" approach to the development of larger and more complex multicellular structures and new materials. This concept starts to become attractive from a technological perspective if we consider theory that a set of common principles underpin a range of macroscopic quantum phenomena [7, 9, 23]. A key objective of future work will be to develop a generic, first principles approach to the development of room temperature macroscopic quantum systems, which can support technologies such as HTSC, CRL and Quantum computing. The primary interest is expected to lie in larger scale, multicellular systems. However, the critical role of quantum vacuum fluctuations in the emergence of mesoscale quantum potentials in structures such as intrinsically disordered proteins and microtubules (see Section 1.4), offers a new approach to the type of work on quantum correlations in dynamical Casimir arrays systems reported by Stassi et al [47], which may have specific applications at the nanometer scale.

As more detailed information on living systems emerges where we can identify specific systems (combinations of biomolecules, ions and local conditions) with the emergence of specific 
morphologies and functionalities, we aim to test our understanding of their influence by replicating conditions in simpler, more controlled inorganic growth experiments. It is anticipated that this approach will offer further insights into the role of the genome, proteome, transcriptome, metabolome, fluxome and electrome in dictating physical processes and their impact on the emergence of structure and function at different scales within biological systems. As we improve our understanding of the mechanisms that underpin biological processes, it should also lead to an improved understanding of the mechanisms and associated diseases that undermine these processes. It is anticipated that this knowledge may be useful in supporting new $a b$ initio approaches to drug design and disease mitigation.

Beyond the work presented in the current paper, the concept of "spin" in biological systems at a multicellular level is worth a more detailed investigation. An example of evidence to support this suggestion is found in a paper by Rossen et al [48], who reported distinct, longrange, well-ordered multicellular vortex patterns in living tissue induced by cell division. The results of the present study add new meaning to such findings. It will be interesting to study the charge density of such systems to determine the extent to which different structures can be influenced and controlled.

It will also be interesting to explore how alternative sources of charge (beyond ions and biopolymers), such as charged nanoparticles can be utilized to manipulate or influence growth processes in organic and inorganic systems. A study to illustrate the principle in biological systems [49], revealed how hydroxyapatite nanoparticles can be used to influence self-organization in the mineralization of bone matrix. With the new theoretical principles developed in the present paper it should be possible to extend this type of work in a more informed and structured approach.

\section{Acknowledgements}

The authors would like to acknowledge the role of the COST office, and in particular COST Action FP1105 for facilitating the collaboration that led to this work.

This research did not receive any specific grant from funding agencies in the public, commercial, or not-for-profit sectors.

\section{Supplementary material.}

The supplementary material contained in this section represents a continuation of Section 2.3.4, offering a mathematical framework to accommodate spin within a macroscopic quantum system. It begins with a description of the biquaternionic velocity field (Eq 24), followed by a series of steps, which lead to derivation of the Pauli equation. 


$$
\begin{gathered}
\mathcal{V}^{\mu}=\frac{1}{2}\left(v_{++}^{\mu}+\tilde{v}_{--}^{\mu}\right)-\frac{i}{2}\left(v_{++}^{\mu}-\tilde{v}_{--}^{\mu}\right) \\
+\left[\frac{1}{2}\left(v_{+-}^{\mu}+\tilde{v}_{-+}^{\mu}\right)-\frac{i}{2}\left(v_{+-}^{\mu}-\tilde{v}_{-+}^{\mu}\right)\right] e_{1} \\
+\left[\frac{1}{2}\left(v_{--}^{\mu}+\tilde{v}_{++}^{\mu}\right)-\frac{i}{2}\left(v_{--}^{\mu}-\tilde{v}_{++}^{\mu}\right)\right] e_{2} \\
+\left[\frac{1}{2}\left(v_{-+}^{\mu}+\tilde{v}_{+-}^{\mu}\right)-\frac{i}{2}\left(\tilde{v}_{-+}^{\mu}+\tilde{v}_{+-}^{\mu}\right)\right] e_{3} .
\end{gathered}
$$

The biquaternionic velocity field corresponds to a biquaternionic derivative operator $\widehat{d} / d s$, which when applied to the position vector $X^{\mu}$, yields the corresponding velocity. Attaching the derivative operator to the velocity in Eq. 24 it reads

$$
\begin{aligned}
& \frac{\widehat{d}}{d s}=\frac{1}{2}\left(\frac{d}{d s}+++\frac{\tilde{d}}{d s}--\right)-\frac{i}{2}\left(\frac{d}{d s}++-\frac{\tilde{d}}{d s}--\right) \\
+ & {\left[\frac{1}{2}\left(\frac{d}{d s}+-+\frac{\tilde{d}}{d s}-+\right)-\frac{i}{2}\left(\frac{d}{d s}+--\frac{\tilde{d}}{d s}-+\right)\right] e_{1} } \\
+ & {\left[\frac{1}{2}\left(\frac{d}{d s}--+\frac{\tilde{d}}{d s}++\right)-\frac{i}{2}\left(\frac{d}{d s}---\frac{\tilde{d}}{d s}++\right)\right] e_{2} } \\
+ & {\left[\frac{1}{2}\left(\frac{d}{d s}-++\frac{\tilde{d}}{d s}+-\right)-\frac{i}{2}\left(\frac{d}{d s}-++\frac{\tilde{d}}{d s}+-\right)\right] e_{3} . }
\end{aligned}
$$

Substituting Eq. (15) and its tilde counterpart into Eq. (25), we obtain the expression for the bi-quaternionic proper-time derivative operator

$$
\frac{\widehat{d}}{d s}=\left\{1+e_{1}+e_{2}+(1-i) e_{3}\right\} \frac{\partial}{\partial s}+\mathcal{V}^{\mu} \partial_{\mu}+i \frac{\lambda}{2} \partial^{\mu} \partial_{\mu}
$$

where the + sign in front of the Dalambertian $\left(\partial^{\mu} \partial_{\mu}\right)$ proceeds from the choice of the metric signature $(+,-,-,-)$.

The term $\partial / \partial s$ in Eq.26 is included for generality. However, since the various physical functions are not explicitly depending on $s$, this term vanishes, leading to a simplified biquaternionic derivative operator

$$
\frac{\widehat{d}}{d s}=\left(\mathcal{V}^{\mu}+i \frac{\lambda}{2} \partial^{\mu}\right) \partial_{\mu} .
$$

Considering the biquaternionic velocity field Eq 24, $i$ denotes the imaginary element used to write the complex components of the biquaternions as sums of a real and an imaginary part 
and the $e_{n}$ (with $n=1,2,3$ ) correspond to the $i, j, k$ imaginary elements normally used to write linear expressions of quaternions as a direct generalization of complex numbers. We can therefore write any biquaternionic wavefunction solution of the Dirac equation, as

$$
\psi=\psi_{0}+\psi_{1} e_{1}+\psi_{2} e_{2}+\psi_{3} e_{3}
$$

whose eight real components we write as,

$$
\psi=\left(\eta_{0}+i \kappa_{0}\right)+\left(\eta_{1}+i \kappa_{1}\right) e_{1}+\left(\eta_{2}+i \kappa_{2}\right) e_{2}+\left(\eta_{3}+i \kappa_{3}\right) e_{3}
$$

Since $\psi$ is assimilated to a Dirac bi-spinor, it must have the same properties. In particular, it must be a unit quaternion, i.e.,

$$
\eta_{0}^{2}+\kappa_{0}^{2}+\eta_{1}^{2}+\kappa_{1}^{2}+\eta_{2}^{2}+\kappa_{2}^{2}+\eta_{3}^{2}+\kappa_{3}^{2}=1 .
$$

Since $\mathcal{V}^{\mu}$ is now biquaternionic, the Lagrange function is also biquaternionic and, therefore, the same is true of the action $\mathcal{S}$. The stationary action principle applied on this action, or equivalently the generalized equivalence principle, as well as the strong covariance principle, all lead to write the equation of motion in the free case (i.e., in the absence of an exterior field) under the form of a differential geodesic equation,

$$
\frac{d \mathcal{V}_{\mu}}{d s}=0
$$

The elementary variation of the action is given by

$$
d \mathcal{S}=\partial_{\mu} \mathcal{S} d x^{\mu}=-m c \mathcal{V}_{\mu} d x^{\mu}
$$

The bi-quaternionic 4-momentum is therefore

$$
\mathcal{P}_{\mu}=m c \mathcal{V}_{\mu}=-\partial_{\mu} \mathcal{S}
$$

We can then introduce a bi-quaternionic wavefunction, as a re-expression of the bi-quaternionic action

$$
\psi^{-1} \partial_{\mu} \psi=\frac{i}{\mathcal{S}_{0}} \partial_{\mu} \mathcal{S}
$$

Since one implicitly uses the quaternionic product between $\psi^{-1}$ and $\partial_{\mu} \psi$. The bi-quaternionic four-velocity is derived from Eq. (33), as

$$
\mathcal{V}_{\mu}=i \frac{S_{0}}{m c} \psi^{-1} \partial_{\mu} \psi
$$


As reported in $[35]^{12}$, by taking linear combinations, one obtains the velocity fields in terms of the wave function components

$$
\begin{aligned}
v_{++}^{\mu}=-\frac{\mathcal{S}_{0}}{m c}\left(\eta_{0} \partial^{\mu} \kappa_{0}+\kappa_{0} \partial^{\mu} \eta_{0}+\eta_{1} \partial^{\mu} \kappa_{1}+\kappa_{1} \partial^{\mu} \eta_{1}+\eta_{2} \partial^{\mu} \kappa_{2}\right. \\
+\kappa_{2} \partial^{\mu} \eta_{2}+\eta_{3} \partial^{\mu} \kappa_{3}+\kappa_{3} \partial^{\mu} \eta_{3}+\eta_{0} \partial^{\mu} \eta_{0}-\kappa_{0} \partial^{\mu} \kappa_{0}+\eta_{1} \partial^{\mu} \eta_{1} \\
\left.-\kappa_{1} \partial^{\mu} \kappa_{1}+\eta_{2} \partial^{\mu} \eta_{2}-\kappa_{2} \partial^{\mu} \kappa_{2}+\eta_{3} \partial^{\mu} \eta_{3}-\kappa_{3} \partial^{\mu} \kappa_{3}\right),
\end{aligned}
$$

$v_{--}^{\mu}=-\frac{\mathcal{S}_{0}}{m c}\left(\eta_{0} \partial^{\mu} \kappa_{0}+\kappa_{0} \partial^{\mu} \eta_{0}+\eta_{1} \partial^{\mu} \kappa_{1}+\kappa_{1} \partial^{\mu} \eta_{1}+\eta_{2} \partial^{\mu} \kappa_{2}\right.$

$$
\begin{aligned}
+\kappa_{2} \partial^{\mu} \eta_{2}+\eta_{3} \partial^{\mu} \kappa_{3} & +\kappa_{3} \partial^{\mu} \eta_{3}-\eta_{0} \partial^{\mu} \eta_{0}+\kappa_{0} \partial^{\mu} \kappa_{0}-\eta_{1} \partial^{\mu} \eta_{1} \\
& \left.+\kappa_{1} \partial^{\mu} \kappa_{1}-\eta_{2} \partial^{\mu} \eta_{2}+\kappa_{2} \partial^{\mu} \kappa_{2}-\eta_{3} \partial^{\mu} \eta_{3}+\kappa_{3} \partial^{\mu} \kappa_{3}\right),
\end{aligned}
$$

$$
\begin{aligned}
v_{+-}^{\mu}=-\frac{\mathcal{S}_{0}}{m c}\left(\eta_{0} \partial^{\mu} \kappa_{1}+\kappa_{0} \partial^{\mu} \eta_{1}-\eta_{1} \partial^{\mu} \kappa_{0}-\kappa_{1} \partial^{\mu} \eta_{0}-\eta_{2} \partial^{\mu} \kappa_{3}\right. \\
-\kappa_{2} \partial^{\mu} \eta_{3}+\eta_{3} \partial^{\mu} \kappa_{2}+\kappa_{3} \partial^{\mu} \eta_{2}+\eta_{0} \partial^{\mu} \eta_{1}-\kappa_{0} \partial^{\mu} \kappa_{1}-\eta_{1} \partial^{\mu} \eta_{0} \\
\left.+\kappa_{1} \partial^{\mu} \kappa_{0}-\eta_{2} \partial^{\mu} \eta_{3}+\kappa_{2} \partial^{\mu} \kappa_{3}+\eta_{3} \partial^{\mu} \eta_{2}-\kappa_{3} \partial^{\mu} \kappa_{2}\right),
\end{aligned}
$$

$$
\begin{aligned}
& v_{-+}^{\mu}=-\frac{\mathcal{S}_{0}}{m c}\left(\eta_{0} \partial^{\mu} \kappa_{1}+\kappa_{0} \partial^{\mu} \eta_{1}-\right. \eta_{1} \partial^{\mu} \kappa_{0}-\kappa_{1} \partial^{\mu} \eta_{0} \\
&-\eta_{2} \partial^{\mu} \kappa_{3}-\kappa_{2} \partial^{\mu} \eta_{3}+\eta_{3} \partial^{\mu} \kappa_{2}+\kappa_{3} \partial^{\mu} \eta_{2}-\eta_{0} \partial^{\mu} \eta_{1}+\kappa_{0} \partial^{\mu} \kappa_{1}+\eta_{1} \partial^{\mu} \eta_{0} \\
&\left.-\kappa_{1} \partial^{\mu} \kappa_{0}+\eta_{2} \partial^{\mu} \eta_{3}-\kappa_{2} \partial^{\mu} \kappa_{3}-\eta_{3} \partial^{\mu} \eta_{2}+\kappa_{3} \partial^{\mu} \kappa_{2}\right),
\end{aligned}
$$

$$
\begin{aligned}
\tilde{v}_{++}^{\mu}=-\frac{\mathcal{S}_{0}}{m c}\left(\eta_{0} \partial^{\mu} \kappa_{2}+\kappa_{0} \partial^{\mu} \eta_{2}+\eta_{1} \partial^{\mu} \kappa_{3}+\kappa_{1} \partial^{\mu} \eta_{3}-\eta_{2} \partial^{\mu} \kappa_{0}\right. \\
-\kappa_{2} \partial^{\mu} \eta_{0}-\eta_{3} \partial^{\mu} \kappa_{1}-\kappa_{3} \partial^{\mu} \eta_{1}+\eta_{0} \partial^{\mu} \eta_{2}-\kappa_{0} \partial^{\mu} \kappa_{2}+\eta_{1} \partial^{\mu} \eta_{3} \\
\left.-\kappa_{1} \partial^{\mu} \kappa_{3}-\eta_{2} \partial^{\mu} \eta_{0}+\kappa_{2} \partial^{\mu} \kappa_{0}-\eta_{3} \partial^{\mu} \eta_{1}+\kappa_{3} \partial^{\mu} \kappa_{1}\right),
\end{aligned}
$$

$$
\begin{aligned}
\tilde{v}_{--}^{\mu}=-\frac{\mathcal{S}_{0}}{m c}\left(\eta_{0} \partial^{\mu} \kappa_{2}+\kappa_{0} \partial^{\mu} \eta_{2}+\eta_{1} \partial^{\mu} \kappa_{3}+\kappa_{1} \partial^{\mu} \eta_{3}-\eta_{2} \partial^{\mu} \kappa_{0}\right. \\
-\kappa_{2} \partial^{\mu} \eta_{0}-\eta_{3} \partial^{\mu} \kappa_{1}-\kappa_{3} \partial^{\mu} \eta_{1}-\eta_{0} \partial^{\mu} \eta_{2}+\kappa_{0} \partial^{\mu} \kappa_{2}-\eta_{1} \partial^{\mu} \eta_{3} \\
\left.+\kappa_{1} \partial^{\mu} \kappa_{3}+\eta_{2} \partial^{\mu} \eta_{0}-\kappa_{2} \partial^{\mu} \kappa_{0}+\eta_{3} \partial^{\mu} \eta_{1}-\kappa_{3} \partial^{\mu} \kappa_{1}\right)
\end{aligned}
$$

$$
\begin{aligned}
\tilde{v}_{+-}^{\mu}=-\frac{\mathcal{S}_{0}}{m c}\left(\eta_{0} \partial^{\mu} \kappa_{3}+\kappa_{0} \partial^{\mu} \eta_{3}-\eta_{1} \partial^{\mu} \kappa_{2}-\kappa_{1} \partial^{\mu} \eta_{2}+\eta_{2} \partial^{\mu} \kappa_{1}\right. \\
+\kappa_{2} \partial^{\mu} \eta_{1}-\eta_{3} \partial^{\mu} \kappa_{0}-\kappa_{3} \partial^{\mu} \eta_{0}+\eta_{0} \partial^{\mu} \eta_{3}-\kappa_{0} \partial^{\mu} \kappa_{3}-\eta_{1} \partial^{\mu} \eta_{2} \\
\left.+\kappa_{1} \partial^{\mu} \kappa_{2}+\eta_{2} \partial^{\mu} \eta_{1}-\kappa_{2} \partial^{\mu} \kappa_{1}-\eta_{3} \partial^{\mu} \eta_{0}+\kappa_{3} \partial^{\mu} \kappa_{0}\right)
\end{aligned}
$$

\footnotetext{
${ }^{12}$ Note that the notation used is that in later work [8]
} 
and

$$
\begin{aligned}
\tilde{v}_{-+}^{\mu}=-\frac{\mathcal{S}_{0}}{m c}\left(\eta_{0} \partial^{\mu} \kappa_{3}+\kappa_{0} \partial^{\mu} \eta_{3}-\eta_{1} \partial^{\mu} \kappa_{2}-\kappa_{1} \partial^{\mu} \eta_{2}+\eta_{2} \partial^{\mu} \kappa_{1}\right. \\
+\kappa_{2} \partial^{\mu} \eta_{1}-\eta_{3} \partial^{\mu} \kappa_{0}-\kappa_{3} \partial^{\mu} \eta_{0}-\eta_{0} \partial^{\mu} \eta_{3}+\kappa_{0} \partial^{\mu} \kappa_{3}+\eta_{1} \partial^{\mu} \eta_{2} \\
\left.-\kappa_{1} \partial^{\mu} \kappa_{2}-\eta_{2} \partial^{\mu} \eta_{1}+\kappa_{2} \partial^{\mu} \kappa_{1}+\eta_{3} \partial^{\mu} \eta_{0}-\kappa_{3} \partial^{\mu} \kappa_{0}\right) .
\end{aligned}
$$

Having described the various components, we can now combine the free motion geodesic equation (Eq.31) and the bi-quaternionic derivative operator Eq.27 to write the equation of motion Eq.44,

$$
\left(\mathcal{V}^{\nu} \partial_{\nu}+i \frac{\lambda}{2} \partial^{\nu} \partial_{\nu}\right) \mathcal{V}_{\mu}=0
$$

which as shown in $[32,33,34]$, underpins the derivation of the Klein-Gordon and Dirac equation.

Since the focus of the present paper lies in the Pauli equation, we refer the reader to [32, $33,34]$ or [8], for a detailed derivation of the Dirac equation. Meanwhile, we consider the relevant, non-relativistic limit of the velocity fields associated with the Dirac equation by decomposing the four component Dirac spinor (Eq 28) into two two-component spinors $\phi$ and $\chi$,

$$
\psi=\left(\begin{array}{l}
\phi \\
\chi
\end{array}\right)
$$

In the quaternionic representation, this amounts to shifting to a symplectic form of $\psi$, where

$$
\phi=\psi_{0}+e_{1} \psi_{1}
$$

and

$$
\chi=\psi_{2}-e_{1} \psi_{3},
$$

so that the spinor reads $\psi=\phi+e_{2} \chi$ since $e_{3}=e_{1} e_{2}$.

In Eq's 46 and 47, the components corresponding to terms with an index 2 or 3 become insignificant in the non-relativistic approximation, where the rest energy $m c^{2}$ becomes dominant and $\chi \ll \phi$. This means that all the tilde velocity fields (Eq's 36-43) introduced from a doubling, due to the breaking of parity (which is specific to the relativistic case) vanish, leaving the four non-zero velocity fields $v_{++}, v_{+-}, v_{-+}$and $v_{--}$.

From this the Pauli spinor $\phi$ is therefore obtained from the Dirac bi-spinor $\psi$, as written in Eq 29 by the correspondence

$$
\phi=\left(\psi_{0}+e_{1} \psi_{1}\right) e^{i m c^{2} t / \hbar}=\left[\left(\eta_{0}+i \kappa_{0}\right)+\left(\eta_{1}+i \kappa_{1}\right) e_{1}\right] e^{i m c^{2} t / \hbar} .
$$


This implies that we obtain the quaternionic velocity corresponding to the non-relativistic limit, first, by neglecting the small components in Eq 24, which gives

$$
\begin{aligned}
\mathcal{V}^{\mu}=\frac{\mathcal{S}_{0}}{m c}\left\{-\left(\eta_{0} \partial^{\mu} \kappa_{0}+\right.\right. & \left.\kappa_{0} \partial^{\mu} \eta_{0}+\eta_{1} \partial^{\mu} \kappa_{1}+\kappa_{1} \partial^{\mu} \eta_{1}\right) \\
& +\left(\eta_{0} \partial^{\mu} \eta_{0}-\kappa_{0} \partial^{\mu} \kappa_{0}+\eta_{1} \partial^{\mu} \eta_{1}-\kappa_{1} \partial^{\mu} \kappa_{1}\right) i \\
& +\left[-\eta_{0} \partial^{\mu} \kappa_{1}-\kappa_{0} \partial^{\mu} \eta_{1}+\eta_{1} \partial^{\mu} \kappa_{0}+\kappa_{1} \partial^{\mu} \eta_{0}\right. \\
& \left.\left.+\left(\eta_{0} \partial^{\mu} \eta_{1}-\kappa_{0} \partial^{\mu} \kappa_{1}-\eta_{1} \partial^{\mu} \eta_{0}+\kappa_{1} \partial^{\mu} \kappa_{0}\right) i\right] e_{1}\right\},
\end{aligned}
$$

then, by distinguishing $\mathcal{V}^{0}$ (which at the non relativistic limit is the velocity of light $c$ ) from the three spatial components, one obtains (after a series of calculations [35]), a threedimensional velocity ( $\mathrm{Eq} 50$ ) derived from the degeneracy of the biquaternionic 4-velocity of the relativistic scenario, in the symplectic form of a real quaternion. One can then construct the correct Pauli equation for the spinor wave function from this velocity field.

$$
\mathcal{V}^{k}=\frac{1}{2}\left(v_{++}^{k}+v_{--}^{k}\right)-\frac{i}{2}\left(v_{++}^{k}-v_{--}^{k}\right)+\left[\frac{1}{2}\left(v_{+-}^{k}+v_{-+}^{k}\right)-\frac{i}{2}\left(v_{+-}^{k}-v_{-+}^{k}\right)\right] e_{1} .
$$

Since $i$ and $e_{1}$ are both roots of -1 , one recovers the standard quaternionic notation by setting $j=e_{1}$ and $k=i e_{1}$.

From the above, we now consider a non-relativistic solution of the Dirac equation (Eq 51)

$$
\left[\gamma^{\mu}\left(i \hbar \partial_{\mu}-e A_{\mu}\right)-m c\right] \psi=0
$$

for an electron of mass $m$ and charge $e$, in an external electromagnetic field $A_{\mu}$, in which the components $\chi \ll \phi$. After some calculations [35] we find a relation between the two components that reads in the Dirac representation

$$
\chi=\frac{\sigma \cdot\left(i \hbar \nabla-\frac{e}{c} \mathbf{A}\right)}{2 m c} \phi .
$$

Since at the non-relativistic limit $\chi \approx(v / c) \ll \phi$, the equation for $\phi$ becomes:

$$
i \hbar \frac{\partial \phi}{\partial t}=\left[\frac{1}{2 m}\left(i \hbar \nabla-\frac{e}{c} \mathbf{A}\right)^{2}-\frac{e \hbar}{2 m c} \sigma \cdot \mathbf{B}+e A_{0}\right] \phi .
$$

which is the Pauli equation for the theory of spin in non-relativistic quantum mechanics, with $i \hbar \nabla$ replacing the momentum operator $\hat{p}$, in accordance with the correspondence principle.

\section{References}

[1] Prigogine. I, George. C, Henin. F. Dynamical and statistical descriptions of n-body systems. Physica, 45, 418-434 (1969). 
[2] Prigogine. I, George, C, Henin, F, Rosenfeld, L. A unified formulation of dynamics and thermodynamics. Chemica Scripta, 4, 5-32 (1973).

[3] Prigogine. I, Petrosky, T, Hasegawa, H, Tasaki, S. Integrability and chaos in classical and quantum mechanics. Chaos, Solitons and Fractals, 1, 3-24 (1991).

[4] Prigogine. I. The end of certainty: time, chaos, and the new laws of nature. New York: The Free Press (1997).

[5] Auffray. C and Nottale. L. Scale relativity theory and integrative systems biology: 1. Founding principles and scale laws. Prog. Biophys. Mol. Biol. 97, 79-114 (2008).

[6] Nottale. L and Auffray. C. Scale relativity theory and integrative systems biology: 2. Macroscopic quantum-type mechanics. Prog. Biophys. Mol. Biol. 97, 115-157 (2008).

[7] Turner. P and Nottale. L. The physical principles underpinning self-organization in plants. Prog. Biophys. Mol. Biol. 123, 48-73 (2017).

[8] Nottale. L. Scale Relativity and Fractal Space-time. A new approach to Unifying Relativity and Quantum Mechanics (2011). Imperial College Press. ISBN 978-1-84816-650-9.

[9] Turner. P and Nottale. L. The origins of macroscopic quantum coherence in high temperature super conductivity. Physica C. 515 15-30 (2015).

[10] Nottale. L. Generalized quantum potentials. J. Phys. A. Math. Theor. 42, 275-306 (2009).

[11] Nottale. L. Fractal Space-Time and microphysics. Towards a theory of scale relativity (1993). World Scientific. ISBN 9810208782.

[12] Zurek. W.H. Decoherence, einselection, and the quantum origins of the classical. Rev. Mod. Phys. 75, 715 (2003)

[13] Schlosshauer. M. The quantum-to-classical transition and decoherence , arXiv:1404.2635v1 [quant-ph] (2014).

[14] Ward. J.J, Sodhi. J.S, McGuffin. LJ, Buxton. B.F, and Jones. D.T. Prediction and Functional Analysis of Native Disorder in Proteins from the Three Kingdoms of Life. Journal of Molecular Biology. 337 Issue 3, 635-645 (2004).

[15] Vattay. G, Salahub D, Csabai. I, Nassimi. A and Kaufmann. S.A Quantum criticality at the origin of life. arXiv:1502.06880v2 (2015).

[16] Zhang. B, Song. W, Pang. P, Zhao. Y, Vattay. G and Lindsay.S. Observation of giant conductance fluctuations in a protein. Nano Futures, Vol 1, No 3.035002 (2017).

[17] Anderson. P. W. Absence of Diffusion in Certain Random Lattices Phys. Rev. 109, 5 (1958).

[18] Vattay. G and Kauffman.S. Quantum biology on the edge of quantum chaos. arXiv:1202.6433v1 [cond-mat.dis-nn] (2012). 
[19] Fratini. M, Poccia. N, Ricci. A, Campi. G, Burghammer. M, Aeppli. G, Bianconi. A. Scale-free structural organization of oxygen interstitials in La2CuO4+y. Nature (london). 466, 841-844 (2010).

[20] Poccia. N, Ricci. A and Bianconi. A. Fractal structure favouring superconductivity at high temperatures in a stack of membranes near a strain quantum critical point. J. Supercond Nov Magn 24, 1195-1200 (2011).

[21] Poccia. N, Ricci. A, Campi. G, Fratini. M, Puri. A, Di Gioacchino. D, Marchelli. A, Reynolds. M, Burghammer. M, Saini. N.L, Aeppli. G and Bianconi. A. Optimum inhomogeneity of local lattice distortions in $\mathrm{La}_{2} \mathrm{CuO}_{4+y}$. Proceedings of the National Academy of Sciences 109 (39), 15685-15690 (2012).

[22] Zaanen. J. High-temperature superconductivity: The benefit of fractal dirt. Nature (london) 466, pages 825-827 (2010).

[23] Turner. P and Nottale. L. A New Ab Initio Approach to the Development of High Temperature Superconducting Materials. J Supercond Nov Magn. 29:3113-3118 (2016). DOI $10.1007 / \mathrm{s} 10948-016-3756-\mathrm{z}$

[24] Derbyshire. P, Menard. D, Green. P, Saalbach. G, Buschmann. H, Lloyd. C.W and Pesquet E. Proteomic analysis of microtubule interacting proteins over the course of xylem tracheary element formation in Arabidopsis. The Plant Cell. 27 2709-2726 (2015).

[25] Nakayama. H, Nakayama. N, Seiki. S, Kojima. M, Sakakibara. H, Sinha. N and Kimuraa S. Regulation of the KNOX-GA Gene Module Induces Heterophyllic Alteration in North American Lake Cress. The Plant Cell 26, 4733-4748 (2014).

[26] Noorduin. W. L, Grinthal. A, Mahadevan. L and Aizenberg.J. Rationally designed complex, hierarchical microarchitectures, Science $340832-837$ (2013).

[27] Ponstingle. H, Krauhs. E, Little.M., and Kempf. T. Complete amino acid sequence of $\alpha$-tubulin from porcine brain.. Proc.Natl.Acad.Sci.USA 78, No.5,pp. 2757-2761 (1981).

[28] Quochi. F, Cordella. Fabrizio, Mura. A, Bongiovanni. Giovanni, Balzer. F, Rubahn. HG. One-Dimensional Random Lasing in a Single Organic Nanofiber. J. Phys. Chem. B 109, 21690-21693 (2005).

[29] Engel. G. S, Calhoun. T.R, Read. E.L, Ahn. T-K, Mancall. T, Cheng. Y-C, Blankenship. R.E, Fleming. G.R. Evidence for wavelike energy transfer through quantum coherence in photosynthetic systems. Nature 446 782-786 (2007).

[30] Collini, E., Wong, C.Y., Wilk, K.E., Curmi, P.M.G., Brumer, P. and Scholes, G.D. Coherently wired light-harvesting in photosynthetic marine algae at ambient temperature. Nature 463 644-647 (2010)

[31] E.M. Gauger, E. Rieper E., J.J.L. Morton, S.C. Ben- jamin, and V. Vedral, Sustained quantum coherence and entanglement in the avian compass Phys. Rev. Lett. 106 (2011), no. 4, 040503. 
[32] Célérier. M.N and Nottale. L. Dirac Equation in scale relativity. arXiv:hep-th/0112213 (2001).

[33] Célérier, M.N. and Nottale, L., A scale-relativistic derivation of the Dirac equation. Electromagnetic Phenomena, T. 3, N.1 (9), 83 (2003). (arXiv: hep-th/0210027)

[34] Célérier M.-N. and Nottale, L. Quantum-classical transition in scale relativity. J. Phys. A 37, 931-955 (2004). (arXiv : quant- ph/0609161).

[35] Célérier. M.N and Nottale. L. The Pauli Equation in scale relativity. J. Phys. A : Math. Gen., 39, 12565-12585. (arXiv : quant-ph/0609107)(2006).

[36] Feynman. R. P and Hibbs. A. R. Quantum Mechanics and Path Integrals. MacGraw-Hill, New York. (1965).

[37] Turner. P, Kowalczyk. M and Reynolds. A. New insights into the micro-fibril architecture of the wood cell wall., COST Action E54 Book. COST Office, Brussels, Belgium. (2011).

[38] Adler S L 1995 Quaternionic Quantum Mechanics and Quantum Fields (New York: Oxford University Press)

[39] Dezael F.X. 2003 Probation Report, Ecole Normale Supérieure (Paris) and Paris Observatory.

[40] Nottale L. Fractals and the quantum theory of space-time. Int. J. Mod. Phys. A A4, 5047-5117 (1989).

[41] Nottale. L and Lehner. T. Turbulence and scale relativity. arXiv : 1807.11902 (2018).

[42] Geesink. H.J.H and Meijer. D. K. F. Quantum Wave Information of Life Revealed: An Algorithm for Electromagnetic Frequencies that Create Stability of Biological Order, with Implications for Brain Function and Consciousness. NeuroQuantology. Vol 14, Issue 1. 106-125 (2016).

[43] Meijer. D. K. F. and Geesink. H.J.H. Phonon Guided Biology: Architecture of Life and Conscious Perception Are Mediated by Toroidal Coupling of Phonon, Photon and Electron Information Fluxes at Discrete Eigenfrequencies. NeuroQuantology. Vol 14, Issue 4. 718-755 (2016).

[44] Melkikh. A. V. and Meijer. D. K. F. On a generalized Levinthal's paradox: The role of long and short range interactions in complex bio-molecular reactions, including protein and DNA folding. Progress in Biophysics and Molecular Biology 132. 57-79 (2018).

[45] Schrödinger. E. What Is Life? The Physical Aspect of the Living Cell. Cambridge University Press, Cambridge (1944).

[46] De Loof. A. The cell's self-generated "electrome": The biophysical essence of the immaterial dimension of Life?. Communicative and Integrative Biology, 9:5, e1197446, DOI: 10.1080/19420889.2016.1197446. (2016). https://doi.org/10.1080/19420889.2016.1197446. 
[47] Stassi. R., De Liberato. S, Garziano. L, Spagnolo. B and Savasta. S. Quantum control and long-range quantum correlations in dynamical Casimir arrays. Physical Review A 92, $013830(2015)$.

[48] Rossen, N. S., Tarp, J. M., Mathiesen, J., Jensen, M. H., and Oddershede, L. B. Longrange ordered vorticity patterns in living tissue induced by cell division. Nature communications 5, 5720 (2014).

[49] Campi, G., Cristofaro, F., Pani, G., Fratini, M., Pascucci, B., Corsetto, P. A., Weinhausen, B., Cedola, A., Rizzo, A. M., Visai, L. and Rea, G. Heterogeneous and selforganizing mineralization of bone matrix promoted by hydroxyapatite nanoparticles. Nanoscale, 9(44), 17274-17283 (2017). 